\title{
SPECIAL FUNCTIONS OF MATRIX ARGUMENT. I: ALGEBRAIC INDUCTION, ZONAL POLYNOMIALS, AND HYPERGEOMETRIC FUNCTIONS
}

\author{
KENNETH I. GROSS AND DONALD ST. P. RICHARDS
}

\begin{abstract}
Hypergeometric functions of matrix argument arise in a diverse range of applications in harmonic analysis, multivariate statistics, quantum physics, molecular chemistry, and number theory. This paper presents a general theory of such functions for real division algebras. These functions, which generalize the classical hypergeometric functions, are defined by infinite series on the space $S=S(n, \mathbf{F})$ of all $n \times n$ Hermitian matrices over the division algebra $\mathbf{F}$. The theory depends intrinsically upon the representation theory of the general linear group $G=G L(n, \mathbf{F})$ of invertible $n \times n$ matrices over $\mathbf{F}$, and the theme of this work is the full exploitation of the inherent group theory. The main technique is the use of the method of "algebraic induction" to realize explicitly the appropriate representations of $G$, to decompose the space of polynomial functions on $S$, and to describe the "zonal polynomials" from which the hypergeometric functions are constructed. Detailed descriptions of the convergence properties of the series expansions are given, and integral representations are provided. Future papers in this series will develop the fine structure of these functions.
\end{abstract}

0. Introduction. We begin a series of articles in which we develop the fine structure of generalized hypergeometric functions of matrix argument. By "fine structure", we allude to the analogues of such classical results as series expansions, integral formulas, asymptotics, differential equations, summation formulas, addition theorems, composition formulas, and recurrence relations. This first paper, in which we simultaneously treat real, complex, and quaternionic analysis, is the result of our desire to present a complete theory of hypergeometric functions of matrix argument over real division algebras, not only as a framework for the body of detailed results to follow in later papers, but also to clarify the representationtheoretic foundation for such a theory.

Although these hypergeometric functions are of interest on purely analytic grounds, they arise in a wide range of applications. Indeed, various classes of

Received by the editors May 19, 1986.

1980 Mathematics Subject Classification (1985 Revision). Primary 22E30, 22E45, 33A75, 43A85, 43A90, 62H10; Secondary 20G20, 32A07, 32M15, 44A10, 62E15.

Key words and phrases. Generalized hypergeometric functions, zonal polynomials, representation theory, algebraic induction, multivariate statistics, general linear group, generalized gamma functions, Pochhammer symbols, Laplace transforms, maximal compact subgroup, invariant polynomials, positive cones, symmetric spaces, Schur functions, special functions of matrix argument.

The first author is on leave from the University of Wyoming.

The second author was partially supported by the National Science Foundation under Grant MCS-8403381, and by the Research Council of the University of North Carolina. 
generalized special functions of matrix argument that are derived from the hypergeometric functions described here, have been studied independently by researchers in harmonic analysis, multivariate statistics, quantum mechanics, and number theory.

In the context of harmonic analysis but motivated by questions in analytic number theory, Bessel functions of a real matrix argument were first introduced by Bochner [1]. Subsequently, Herz [15] treated those Bessel functions in greater detail and also defined hypergeometric functions of matrix argument inductively through iterative applications of Laplace and inverse Laplace transforms. Numbertheoretic investigations of Siegel [28] and Selberg [26], led Gindikin [6] to introduce generalizations of the Gaussian hypergeometric functions, defined by Eulertype integrals, associated with homogeneous cones and analysis on Siegel domains. Number-theoretic applications continue, for example, in recent work of Terras [31] and Shimura [27]. In multivariate statistical theory, series expansions of Herz' hypergeometric functions were developed by James, Constantine, and others to study certain invariant statistical distributions. We refer to James [19], Muirhead [23] and Takemura [29] for extensive surveys of the statistical literature. Although series expansions for hypergeometric functions appear in the literature for both the real and complex fields $[\mathbf{1 9}]$, there seem to be no analogous results in the quaternionic case.

In the study of infinite-dimensional representation theory, .Gross and Kunze $[\mathbf{8 ,}, \mathbf{9}]$ developed a theory of operator-valued Bessel functions of matrix argument over real division algebras, which included as special cases the Bessel functions considered by Herz and James. These operator-valued Bessel functions arose group-theoretically from the Peter-Weyl theory applied to compact group actions on Euclidean spaces. In the context of quantum mechanics, Louck and Biedenharn [22] investigated a generalization of Gauss' ${ }_{2} F_{1}$ hypergeometric function that coincides with a special case of James' hypergeometric functions [19]. By means of the "contraction principle" utilized in quantum physics, Holman [16] derived series expansions for the above-mentioned operator-valued Bessel functions on complex $2 \times 2$ matrix space. More recently, it has been shown by Richards [24] that integrals arising in Eichinger's work [4] in molecular chemistry are related to the Bessel functions of Herz.

The above remarks, while illustrating the widespread applicability of the hypergeometric functions of matrix argument, also speak to an isolation whereby researchers in various fields are essentially unaware of each other's work. In part, that accounts for the fact that - except for analogues of Gauss' summation formula in $[\mathbf{6}]$ and $[\mathbf{1 9}]$ - the literature fails to address the fine structure of these functions.

The primary thrust of our work in this paper is the full exploitation of the inherent symmetries in order to blend earlier approaches into a general theory. The key idea is to use the method of algebraic induction [10] to construct explicitly the appropriate representations.

The hybrid nature of this work is manifested in our presentation. For example, whereas some aspects of our approach will be readily recognizable to statisticians, other components will be familiar to harmonic analysts. With this in mind, we have adopted an informal expository style, while still adhering to mathematical rigor. This formulation will allow the statistician or physical scientist to restrict 
the context to the real or complex field, as appropriate, and to suppress the detail of proofs if desired. On the other hand, the reader familiar with Lie theory will see that our methods have more general applicability to symmetric spaces.

With the above as background and motivation, we give an overview of our results.

Let $\mathbf{F}$ denote a real finite-dimensional division algebra, equipped with its usual conjugation, let $G=G L(n, \mathbf{F})$ be the group of all invertible $n \times n$ matrices over $\mathbf{F}$, and let $S=S(n, \mathbf{F})$ be the space of all Hermitian $n \times n$ matrices $s$ over $\mathbf{F}$ under the action $s \rightarrow a^{*} s a$ of $G$. Hypergeometric functions of matrix argument are certain real-analytic functions on $S$ which are invariant under the maximal compact subgroup $K$ of $G$. The study of any $K$-invariant real-analytic function on $S$, and the hypergeometric functions in particular, rests intrinsically on the representation theory of $G$. In fact, the definition of such functions as power series requires the decomposition of the algebra $P(S)$ of all polynomial functions on $S$ under the action of $G$. The importance of this problem, especially for complex analysis in several variables, has attracted the attention of many authors. Hua [18] showed that the decomposition of $P(S)$ is multiplicity free, and he determined which irreducible representations of $G$ appear. Hua's work was later formulated Lie theoretically and extended by Schmid [25], Takeuchi [30], and Johnson [20].

Our contribution here is to provide a simple, intrinsic, global construction of the irreducible subspaces of $P(S)$. There are two main ingredients. First, for each $n$-tuple $m=\left(m_{1}, \ldots, m_{n}\right)$ of integers such that

$$
m_{1} \geq m_{2} \geq \cdots \geq m_{n} \geq 0
$$

we utilize the method of algebraic induction [10] to construct an irreducible polynomial representation $\pi_{2 m}$ of $G$ with signature $2 m$ given by (2.6.1), acting by right translation in a space $p^{2 m}(G)$ of "covariantly transforming" polynomials on $G$ (cf. (2.8)). Secondly, we apply Weyl's classical invariant theory [34] to show that the spherical transform $\phi \rightarrow p$ defined by

$$
p\left(x^{*} x\right)=\int_{K} \phi(k x) d k
$$

is a bijection of $P^{2 m}(G)$ with an irreducible subspace $P^{m}(S)$, of polynomial functions on $S$ homogeneous of degree $|m|=m_{1}+\cdots+m_{n}$. Now, Hua's results can be formulated as the explicit multiplicity-free decomposition

$$
P(S)=\sum_{m} \bigoplus^{p^{m}}(S)
$$

Our methodology emphasizes a positivity condition on representations, in terms of which (3) has a different interpretation. We say that an irreducible polynomial representation of $G$ is totally positive if there exists a nonzero real-valued matrix entry that is nonnegative. Let $\pi$ be an irreducible polynomial representation of $G$. Then the following are equivalent:

(i) $\pi$ is totally positive.

(ii) $\pi$ has a $K$-invariant vector.

(iii) $\pi$ has signature $2 m$ of the form (2.6.1).

(iv) $\pi$ is equivalent to $\pi_{2 m}$ for some $m$.

(v) $\pi$ appears in $\mathrm{P}(S)$. 
The decomposition (3) implies the existence of the required $K$-invariant polynomials. In each irreducible subspace $\rho^{m}(S)$ there is a nonzero polynomial $Z_{m}$, unique up to scalar multiples, that is $K$-invariant. Thus, the algebra $P(S)^{K}$ of all $K$-invariant polynomials on $S$ is spanned by the polynomials $Z_{m}$, and any realanalytic $K$-invariant function $f$ has a power series expansion

$$
f(s)=\sum_{m} c_{m} Z_{m}(s)
$$

We refer to $Z_{m}$ as the zonal polynomial on $S$ of weight $m$. The normalization of $Z_{m}$ is fixed by the condition (Lemma 5.2)

$$
(\operatorname{tr} s)^{d}=\sum_{|m|=d} Z_{m}(s)
$$

or equivalently,

$$
e^{\operatorname{tr} s}=\sum_{d=0}^{\infty} \frac{1}{d !} \sum_{|m|=d} Z_{m}(s)
$$

With the above machinery in place, we can now describe the hypergeometric functions. Let $\nu$ denote the real dimension of $\mathbf{F}$. Fix complex numbers $\alpha_{1}, \ldots, \alpha_{p}$ and $\beta_{1}, \ldots, \beta_{q}$, and for all $1 \leq i \leq q$ and $1 \leq j \leq n$ do not allow $-\beta_{i}+(j-1) \nu / 2$ to be a nonnegative integer. Then the hypergeometric function ${ }_{p} F_{q}$ is defined to be the real-analytic function on $S$ given by the series

$$
{ }_{p} F_{q}\left(\alpha_{1}, \ldots, \alpha_{p} ; \beta_{1}, \ldots, \beta_{q} ; s\right)=\sum_{d=0}^{\infty} \sum_{|m|=d} \frac{\left[\alpha_{1}\right]_{m} \cdots\left[\alpha_{p}\right]_{m}}{\left[\beta_{1}\right]_{m} \cdots\left[\beta_{q}\right]_{m}} \frac{Z_{m}(s)}{d !}
$$

When $n=1$ and $\mathbf{F}$ is the real field, this reduces to the classical hypergeometric series. The coefficients in (7) are defined in terms of a generalization $[\alpha]_{m}$ of the classical Pochhammer symbol appropriate to the division algebra $\mathbf{F}$ and the weight $m$. More specifically, the generalized gamma function of weight $m$ for the space $S$ is defined by

$$
\Gamma_{n}(\alpha, m)=\int_{P} e^{-\operatorname{tr} r} \Delta(r)^{\alpha} q_{m}(r) d_{*} r
$$

where $P$ is the cone of positive matrices in $S, \Delta$ is essentially the determinant (cf. (1.2)), $q_{m}$ is the highest weight vector in $P_{m}(S)$, and $d_{*} r$ is invariant measure on $P$. Then in analogy to the functional equation for the classical gamma function,

$$
[\alpha]_{m}=\frac{\Gamma_{n}(\alpha, m)}{\Gamma_{n}(\alpha)}=\prod_{j=1}^{n}\left(\alpha-\frac{1}{2}(j-1) \nu\right)_{m_{j}}
$$

where $\Gamma_{n}(\alpha)$ is the special case of $(8)$ in which $m=(0, \ldots, 0)$ corresponds to the identity representation of $G$, and $(\alpha)_{j}=\alpha(\alpha+1) \cdots(\alpha+j-1)$ is the standard Pochhammer symbol. We give a complete description of the convergence of the series (7), and we obtain integral representations for the hypergeometric functions.

We conclude this introduction with a summary of the organization of the paper. In $\S 1$ we compile the properties of the group $G$ that enter into this work. 
$\S 2$ is devoted to the construction of the irreducible representations $\pi_{2 m}$ and the structure of the spaces $P^{2 m}(G)$. These are the representations of $G$ that appear in $P(S)$.

The decomposition of the space $I(G)$ of left $K$-invariant polynomials on $G$ appears in $\S 3$.

In $\S 4$ we convert the results of the preceding section into the decomposition of $P(S)$, and we define the zonal polynomials.

In $\S 5$ we derive a number of properties of the zonal polynomials that are needed for the study of the hypergeometric functions. These include a Laplace transform formula (Theorem 5.9) and an Euler-type integral for $Z_{m}$ (Corollary 5.10). The generalized gamma function also appears in this section.

$\S 6$ contains our treatment of the generalized hypergeometric functions. We give the convergence properties of the hypergeometric series in Theorem 6.3. Integral representations appear in Proposition 6.11 and Theorem 6.13.

Throughout the paper, we utilize script $P$ to indicate an algebra of polynomial functions. Subscripts such as $d$ or $2 d$ are reserved for degree of homogeneity, and superscripts such as $m$ or $2 m$ index irreducible subspaces.

1. The general linear group $G=G L(n, \mathbf{F})$. We consider the general linear group $G=G L(n, \mathbf{F})$ of all $n \times n$ matrices over $\mathbf{F}$, where $\mathbf{F}$ is either the real field $\mathbf{R}$, the complex field $\mathbf{C}$ regarded as a two-dimensional real algebra, or the four-dimensional real (noncommutative) division algebra $\mathbf{H}$ of quaternions. In this section, which is ancillary in nature, we collect some basic structural properties of $G$. In particular, the standard bitriangular structure in (1.4) is central to the representation theory of the next section.

1.1 The underlying field $\mathbf{F}$. Throughout, $\mathbf{F}$ denotes a real finite dimensional division algebra. Thus, $\mathbf{F}$ is the real field $\mathbf{R}$, the complex field $\mathbf{C}$, or the quaternion algebra $\mathbf{H}$. We equip $\mathbf{F}$ with its usual conjugation $t \rightarrow \bar{t}$, and set $\operatorname{Re}(t)=(t+\bar{t}) / 2$ and $|t|=(t \bar{t})^{1 / 2}$. Denote by $\nu$ the real dimension of $\mathbf{F}$; so $\nu=1,2$, or 4 according as $\mathbf{F}=\mathbf{R}, \mathbf{C}$, or $\mathbf{H}$.

We write complex numbers in the standard way as $t=t_{1}+t_{2} i$ with $t_{1}$ and $t_{2}$ real. For the quaternions, we realize the elements of $\mathbf{H}$ as $2 \times 2$ complex matrices of the form

$$
t=\left(\begin{array}{cc}
z & w \\
-\bar{w} & \bar{z}
\end{array}\right)
$$

Hence, $\mathbf{H}$ is a real subalgebra of $\mathbf{C}^{2 \times 2}$, and conjugation in $\mathbf{H}$ is the adjoint operation in $\mathbf{C}^{2 \times 2}$. That is,

$$
\bar{t}=\left(\begin{array}{cc}
\bar{z} & -w \\
\bar{w} & z
\end{array}\right)
$$

for $t \in \mathbf{H}$. When $\mathbf{H}$ is viewed as a real vector space and it is necessary to introduce a basis (cf. (4.2.2)), we write a quaternion in the standard way as $t=t_{1} 1+t_{2} i+$ $t_{3} j+t_{4} k$ with $t_{1}, \ldots, t_{4}$ real, where 1 is the $2 \times 2$ identity matrix,

$$
i=\left(\begin{array}{cc}
i & 0 \\
0 & -i
\end{array}\right), \quad j=\left(\begin{array}{cc}
0 & 1 \\
-1 & 0
\end{array}\right), \quad \text { and } \quad k=\left(\begin{array}{cc}
0 & i \\
i & 0
\end{array}\right) \text {. }
$$

Note that $t \in \mathbf{R}$ means $t=\bar{t}$, and we identify $t$ with $t 1 \in \mathbf{H}$. 
1.2 The function $\Delta$. Throughout, we fix a positive integer $n$, and we set $N=\nu n^{2}$. Let $\mathbf{F}^{n \times n}$ be the set of all $n \times n$ matrices over $\mathbf{F}$, regarded as a real algebra with involution $x \rightarrow x^{*}$, where $x^{*}=\bar{x}^{t}$. The dimension of $\mathbf{F}^{n \times n}$ over $\mathbf{R}$ is $N$. In the quaternionic case, we stress that an $n \times n$ matrix over $\mathbf{H}$ is a $2 n \times 2 n$ complex matrix.

Let det denote the determinant function on $\mathbf{F}^{n \times n}$. The determinant of a quaternionic matrix is necessarily nonnegative (cf. (1.5)). Set

$$
\eta= \begin{cases}1 & \text { if } \mathbf{F}=\mathbf{R} \text { or } \mathbf{C} \\ 1 / 2 & \text { if } \mathbf{F}=\mathbf{H}\end{cases}
$$

and define the function $\Delta$ by

$$
\Delta(x)=(\operatorname{det} x)^{\eta}
$$

for $x \in \mathbf{F}^{n \times n}$. For our purposes it is more convenient to use the function $\Delta$ than the determinant. This function $\Delta$ is well defined and continuous on $\mathbf{F}^{n \times n}$, but it is a polynomial only when $\mathbf{F}=\mathbf{R}$ or $\mathbf{F}=\mathbf{C}$. However, irrespective of the nature of $\mathbf{F}$, the function $|\Delta|^{2}$ is a nonnegative real-valued polynomial on $\mathbf{F}^{n \times n}$.

1.3 The general linear group $G$. Denote by $G=G L(n, \mathbf{F})$ the group of all invertible elements of $\mathbf{F}^{n \times n}$. Alternatively, the group $G$ can be characterized as all $n \times n$ matrices $x$ over $\mathbf{F}$ such that $\Delta(x) \neq 0$. We call $G$ the $n \times n$ general linear group over $\mathbf{F}$, and we regard it throughout as a real Lie group.

In this paper we will treat the three cases of the real, complex, and quaternionic fields simultaneously without recourse to the specific nature of the field. However, a few observations concerning the nature of the individual cases are appropriate to keep in mind. First, we emphasize for the real field that $G$ is the full general linear group $G L(n, \mathbf{R})$, which is not connected. Next, since the complex general linear group $G L(n, \mathbf{C})$ is regarded as a real group, we deal with real-analytic functions on $G L(n, \mathbf{C})$ rather than holomorphic functions, and with polynomials of real argument rather than complex argument. Finally, we note that the quaternionic general linear group $G L(n, \mathbf{H})$ is a real Lie group of $2 n \times 2 n$ complex matrices.

Throughout, we fix Lebesgue measure $d x$ on $\mathbf{F}^{n \times n}$, normalized such that the integral of the Gaussian $x \rightarrow \exp \left[-\operatorname{tr}\left(x^{*} x\right)\right]$ is $\pi^{N / 2}$. Then, from $\S 5$ of [8],

$$
d_{*} x=|\Delta(x)|^{-\nu n} d x
$$

is Haar measure on the group $G$.

The maximal compact subgroup $K$ of $G$ consists of all matrices $k$ in $\mathbf{F}^{n \times n}$ such that $k k^{*}=1$. Thus, $K$ is the real orthogonal group $O(n)$, the unitary group $\mathcal{U}(n)$, or the compact symplectic group $S p(n)$ according as $\mathbf{F}=\mathbf{R}, \mathbf{C}$, or $\mathbf{H}$.

We note in passing that $S p(1)=S U(2)$, the special unitary group of $2 \times 2$ unitary matrices of determinant 1 , and the group $\mathbf{H}^{\times}=G L(1, \mathbf{H})$ of nonzero quaternions is the direct product of the group $\mathbf{R}^{+}$of positive reals with $S U(2)$.

1.4 The standard bitriangular structure of $G$. Let $C=C_{n}$ be the diagonal subgroup of $G$ consisting of all $n \times n$ matrices $c=\operatorname{diag}\left(c_{1}, \ldots, c_{n}\right)$ where $c_{j}$ are invertible elements of $\mathbf{F}, j=1, \ldots, n$. Let $U=U_{n}$ be the subgroup of all upper 
triangular matrices of the form

$$
u=\left(\begin{array}{ccccc}
1 & u_{12} & u_{13} & \ldots & u_{1 n} \\
0 & 1 & u_{23} & \ldots & u_{2 n} \\
0 & 0 & 1 & \ldots & u_{3 n} \\
\vdots & \vdots & \vdots & & \vdots \\
0 & 0 & 0 & \ldots & 1
\end{array}\right)
$$

with $u_{i j} \in \mathbf{F}$ for $1 \leq i<j \leq n$; and let $V=V_{n}$ be the opposed lower triangular subgroup $V=U^{t}$. Note that if $\mathbf{F}=\mathbf{R}$ or $\mathbf{C}$ then $C$ is abelian (the Cartan subgroup of $G$ ), and $U$ and $V$ are the full upper and lower triangular unipotent subgroups of $G$, respectively. When $\mathbf{F}=\mathbf{H}$, however, $C$ is nonabelian (and isomorphic to the direct product of $n$ copies of $\left.\mathbf{H}^{\times}\right)$. We refer to the triple $(U, C, V)$ as the standard bitriangular structure for $G$. It is a bitriangular structure for $G$ in the sense of [10]. In particular, $C$ normalizes both $U$ and $V$, and the product $(u, c, v) \rightarrow v c u$ is a bianalytic map of $U \times C \times V$ onto the dense open subset of $G$ consisting of all matrices $x$ such that

$$
\prod_{j=1}^{n} \Delta_{j}(x) \neq 0
$$

where

$$
\Delta_{j}(x)=\Delta\left(\begin{array}{ccc}
x_{11} & \ldots & x_{1 j} \\
\vdots & & \vdots \\
x_{j 1} & \ldots & x_{j j}
\end{array}\right)
$$

with $\Delta$ given by (1.2.2).

1.5 REMARK. Note that for the quaternionic case, the standard bitriangular structure for $G L(n, \mathbf{H})$ yields the fact that $\operatorname{det} x \geq 0$ for all $x \in \mathbf{H}^{n \times n}$. Indeed, if $x \in G$ is of the form $x=v c u$, as above, then $\operatorname{det} x=\left|c_{1} \cdots c_{n}\right|^{2}>0$. As such elements are dense in $\mathbf{F}^{n \times n}$, the determinant is nonnegative for all $x \in \mathbf{F}^{n \times n}$.

1.6 The cone $P=P(n, \mathbf{F})$. Let $P=P(n, \mathbf{F})$ be the cone of positive $n \times n$ matrices $r$ over $\mathbf{F}$. Thus, $P$ consists of all matrices $r=x^{*} x$ with $x$ in $G$. Let $B$ be the subgroup of $G$ consisting of upper triangular matrices with positive entries on the diagonal. That is, $B=U C^{+}$where $U$ is given by (1.4.1) and $C^{+}=C \cap P$ is the subgroup of $G$ composed of diagonal matrices $c=\operatorname{diag}\left(c_{1}, \ldots, c_{n}\right)$ with the entries $c_{j}$ being real and positive. We write elements of $B$ as $b=u c$, where $u \in U$ and $c \in C^{+}$. Then the mapping $b \rightarrow r=b^{*} b$ is a diffeomorphism of $B$ with $P$. Let $d_{*} r$ denote the measure on $P$ invariant under the action $r \rightarrow a^{*} r a$ of $G$. We normalize this measure as follows. Let

$$
d u=\prod_{i<j} d u_{i j} \quad \text { and } \quad d_{*} c=\prod_{i=1}^{n} c_{i}^{-1} d c_{i}
$$

be Lebesgue measure on $U$ and invariant measure on $C^{+}$, respectively. Then

$$
d_{*} r=2^{n} d u d_{*} c
$$

The normalizing factor $2^{n}$ arises as follows. Let

$$
d s=\prod_{1 \leq i \leq j \leq n} d s_{i j}
$$


be Lebesgue measure on $S$ in Cartesian coordinates, and set

$$
\theta=\frac{1}{2}[(n-1) \nu+2] \text {. }
$$

The straightforward calculation of the Jacobian for the coordinate change $(c, u) \rightarrow$ $r=c^{*} u^{*} u c$, shows that

$$
d_{*} r=\Delta(r)^{-\theta} d r
$$

where $d r$ is the restriction of Lebesgue measure on $S$ to $P$.

1.7 Polar coordinates on $G$. The homogeneous space $K \backslash G$ of right cosets $K x$ may be identified with $P$ by means of the mapping $k x \rightarrow r=x^{*} x$ that intertwines the (right) action of $G$ on $K \backslash G$ with the action $r \rightarrow a^{*} r a$ of $G$ on $P$. Indeed, any matrix $x \in G$ has a unique decomposition $x=k r^{1 / 2}$ into polar coordinates where $k \in K$ and $r^{1 / 2}$ is the unique positive square root of $r=x^{*} x$. From $\S 5$ of [8], relative to polar coordinates, Haar measure on $G$ splits as

$$
d_{*} x=\beta_{n} d k d_{*} r
$$

where $d k$ is Haar measure on $K$ normalized by $\operatorname{vol}(K)=1$. Here, $\beta_{n}$ is a positive constant which, as we will see in (5.8), has the exact value

$$
\beta_{n}=\pi^{\nu n(n+1) / 4} / \prod_{j=1}^{n} \Gamma(\nu(n-j+1) / 2) .
$$

2. Totally positive representations. This section contains the representation theoretic basis for the analysis to follow. Rather than give a systematic exposition of the representation theory of $G$, our goal is to develop the theory only to the extent necessary to give a unified and systematic presentation of our work on zonal polynomials and hypergeometric function on $\mathbf{F}^{n \times n}$. In particular, our analysis requires only a certain set of irreducible representations, denoted $\pi_{2 m}$, that are polynomial and totally positive.

2.1 Polynomials on $G$. A polynomial function on $G$ is just the restriction of a polynomial on the real vector space $\mathbf{F}^{n \times n}$. Since $G$ is an open subset of $\mathbf{F}^{n \times n}$, a polynomial function on $G$ extends uniquely to a polynomial on $\mathbf{F}^{n \times n}$, and conversely a polynomial on $\mathbf{F}^{n \times n}$ has a unique restriction to a polynomial on $G$. Let $P(G)$ denote the algebra of all polynomials on $G$, graded by degree, and let $P_{d}(G)$ be the subspace of polynomials homogeneous of degree $d$. We let $G$ act from the right, and define the right regular representation of $G$ on $P(G)$ by

$$
(R(a) \phi)(x)=\phi(x a)
$$

for all $a \in G$ and $\phi \in \mathcal{P}(G)$. The subspaces $\mathcal{P}_{d}(G)$ are all finite-dimensional and invariant under $R$, and

$$
\operatorname{dim} P_{d}(G)=\left(\begin{array}{c}
N+d-1 \\
N-1
\end{array}\right) .
$$

2.2 The differentiation inner product on $P(G)$. The inner product on $P(G)$ to be defined below is well known for real Euclidean spaces, so we regard $\mathbf{F}^{n \times n}$ as $\mathbf{R}^{N}$. Thus, a polynomial on $\mathbf{F}^{n \times n}$ is a complex-valued function of the $N=\nu n^{2}$ real coordinates $X_{1}, \ldots X_{N}$. Specifically, each of these coordinate functions is of the form $\left(x_{i j}\right)_{\varsigma}$ where $x \in \mathbf{F}^{n \times n}$ and we enumerate $(i, j, \varsigma)$ lexicographically as $i$ 
and $j$ vary from 1 to $n$ and $\zeta$ from 1 to $\nu$. We write $X=\left(X_{1}, \ldots, X_{N}\right) \in \mathbf{R}^{N}$. For a multi-index $\alpha=\left(\alpha_{1}, \ldots, \alpha_{N}\right)$ we set

$$
X^{\alpha}=X_{1}^{\alpha_{1}} \cdots X_{N}^{\alpha_{N}} \quad \text { and } \quad \alpha !=\left(\alpha_{1} !\right) \cdots\left(\alpha_{N} !\right)
$$

and we put $\phi \in P(G)$ in the standard form

$$
\phi(x)=\sum_{\alpha} a_{\alpha} X^{\alpha}
$$

where the $a_{\alpha}$ are complex coefficients. Define $\tilde{\phi} \in \mathcal{P}(G)$ by replacing $a_{\alpha}$ in (2) by its complex conjugate $\bar{a}_{\alpha}$. The mapping $\phi \rightarrow \tilde{\phi}$ is a conjugate-linear automorphism of $P(G)$ that preserves homogeneity degree and is the identity on real-valued functions. Next, for any polynomial $\phi$ the corresponding differential operator $D(\phi)$ is obtained by replacing each coordinate function $X_{i}$ by the corresponding partial derivative $\partial / \partial X_{i}$. Then the formula

$$
\langle\phi \mid \psi\rangle=(D(\tilde{\psi}) \phi)(0)
$$

defines an inner product on $\mathcal{P}(G)$. Indeed, if $\phi$ and $\psi$ are written in standard form (2) with coefficients $a_{\alpha}$ and $b_{\alpha}$, respectively, then

$$
\langle\phi \mid \psi\rangle=\sum_{\alpha}(\alpha !) a_{\alpha} \bar{b}_{\alpha}
$$

Note that the subspaces $\mathcal{P}_{d}(G)$ are mutually orthogonal and span $\mathcal{P}(G)$.

We relate the inner product to the representation theory. For each $a \in G$

$$
\left\langle R(a) \phi \mid R\left(a^{\vee}\right) \psi\right\rangle=\langle\phi \mid \psi\rangle
$$

for all $\phi$ and $\psi$ in $P(G)$, where

$$
a^{\vee}=\left(a^{*}\right)^{-1} .
$$

Property (5) can be rephrased as follows. Relative to this inner product on $P(G)$,

$$
R\left(a^{*}\right)=R(a)^{*}
$$

for all $a \in G$. The proof of (5) follows immediately from (3) together with the property

$$
R(a) D(\tilde{\phi}) R\left(a^{-1}\right)=D\left(\left(R\left(a^{\vee}\right) \phi\right)^{\sim}\right)
$$

for $a \in G$ and $\phi \in \mathcal{P}(G)$. This last formula is proved in $[\mathbf{3 3}]$ for the case $\mathbf{F}=\mathbf{R}$. It follows for general $\mathbf{F}$ by the observation that the mapping $x \rightarrow x a^{*}$ on $\mathbf{F}^{n \times n}$ corresponds to the linear transformation $X \rightarrow X A^{t}$ on $\mathbf{R}^{N}$, where $A$ is the $N \times N$ real matrix corresponding to the $n \times n$ matrix $a$ over $\mathbf{F}$.

As a special case of (5),

$$
\langle R(k) \phi \mid R(k) \psi\rangle=\langle\phi \mid \psi\rangle
$$

for all $k$ in the subgroup $K$, so the restriction of $R$ to the maximal compact subgroup is unitary.

2.3 Representations. For the remainder of this section, we use the term representation to mean a finite-dimensional representation of $G$ which is rational in that all the matrix entries are quotients of polynomials in the $N=\nu n^{2}$ real entry functions of $\mathbf{F}^{n \times n}$. For the reader who is less familiar with representation theory, 
we remark that the rational structure of such a representation is quite rigidly determined. (See, e.g., $[\mathbf{9}, \S 3]$ or $[\mathbf{1 0},(6.18)]$.) A representation is said to be polynomial if the matrix entries are all polynomials in the $N$ real matrix entries. Then for any representation $T$ of $G$, rational according to the preceding definition, there exist nonnegative integers $k_{1}, k_{2}$ such that the representation $x \rightarrow(\operatorname{det} x)^{k_{1}}(\overline{\operatorname{det} x})^{k_{2}} T(x)$ is polynomial. Of course, when $\mathbf{F}=\mathbf{R}$ or $\mathbf{H}$ we may take $k_{2}=0$. In particular, all representations of $G$ are determined from the polynomial representations. Since representations are assumed rational, they are completely reducible. We are led, therefore, to study the irreducible representations of $G$.

2.4 The signature of an irreducible representation. Let $\pi$ denote the generic irreducible representation of $G$.

(1) Let $\mathbf{F}=\mathbf{R}$. The representations $\pi=\pi_{m}$ of $G L(n, \mathbf{R})$ are parametrized by $n$-tuples $m=\left(m_{1}, \ldots, m_{n}\right)$ of integers such that $m_{1} \geq \cdots \geq m_{n}$.

(2) Let $\mathbf{F}=\mathbf{C}$. The representations $\pi=\pi_{m \times l}$ of $G L(n, \mathbf{C})$ are parametrized by $n$-tuples $m=\left(m_{1}, \ldots, m_{n}\right)$ and $l=\left(l_{1}, \ldots, l_{n}\right)$ of integers such that $m_{1} \geq \cdots \geq m_{n}$ and $l_{1} \geq \cdots \geq l_{n}$.

(3) Let $\mathbf{F}=\mathbf{H}$. The representations $\pi=\pi_{M}$ of $G L(n, \mathbf{H})$ are parametrized by $2 n$-tuples $M=\left(M_{1}, \ldots, M_{2 n}\right)$ of integers such that $M_{1} \geq \cdots \geq M_{2 n}$.

We refer to the index $m, m \times l$, or $M$ as the signature of the corresponding representation $\pi_{m}, \pi_{m \times l}$, or $\pi_{M}$ respectively. Our interest lies in the irreducible representations that are polynomial. The irreducible representation $\pi_{m}$ of $G L(n, \mathbf{R})$ is polynomial if and only if $m_{n} \geq 0$; the irreducible representation $\pi_{m \times l}$ of $G L(n, \mathbf{C})$ is polynomial if and only if both $m_{n} \geq 0$ and $l_{n} \geq 0$; and the irreducible representation $\pi_{M}$ of $G L(n, \mathbf{H})$ is polynomial if and only if $M_{2 n} \geq 0$.

2.5 REMARK. In the remainder of this section we construct irreducible representations of $G$ in spaces of polynomials on $G$. We do this, not for all the irreducible representations, but only those irreducible representations that enter into the study of zonal polynomials and hypergeometric functions. For more detail concerning signatures see $\S 3$ of $[\mathbf{9}]$.

2.6 The signatures $2 m$. Certain signatures are fundamental to this work. We denote them by

$$
2 m= \begin{cases}\left(2 m_{1}, \ldots, 2 m_{n}\right) & \text { when } \mathbf{F}=\mathbf{R} \\ \left(m_{1}, \ldots, m_{n}\right) \times\left(m_{1}, \ldots, m_{n}\right) & \text { when } \mathbf{F}=\mathbf{C} \\ \left(m_{1}, m_{1}, m_{2}, m_{2}, \ldots, m_{n}, m_{n}\right) & \text { when } \mathbf{F}=\mathbf{H}\end{cases}
$$

where $m=\left(m_{1}, \ldots, m_{n}\right)$ is an $n$-tuple of integers such that

$$
m_{1} \geq m_{2} \geq \cdots \geq m_{n} \geq 0 \text {. }
$$

The representations $\pi_{2 m}$ are all polynomials.

2.7 Definition. An irreducible polynomial representation of $G$ is said to be totally positive if there exists a nonzero real-valued matrix entry that is nonnegative. Note that if a representation $\pi$ in a space $V$ is totally positive, then there exists an open convex cone in $V$, which is invariant under $\pi$. In (2.10) the representations $\pi_{2 m}$ are shown to be totally positive. In the next section we will see that these are all the totally positive representations of $G$.

2.8 Covariantly transforming polynomials. Let $(U, C, V)$ be the standard bitriangular structure given in (1.4). For each $n$-tuple $m=\left(m_{1}, \ldots, m_{n}\right)$ of integers 
satisfying (2.6.2), denote by $\mu_{2 m}$ the character of $C$ defined by

$$
\mu_{2 m}(c)=\left|c_{1}\right|^{2 m_{1}}\left|c_{2}\right|^{2 m_{2}} \cdots\left|c_{n}\right|^{2 m_{n}}
$$

for $c=\operatorname{diag}\left(c_{1}, \ldots, c_{n}\right) \in C$. Let $P^{2 m}(G)$ be the subspace of $P(G)$ consisting of all polynomials $\phi$ such that

$$
\phi(v c x)=\mu_{2 m}(c) \phi(x)
$$

for all $(v, c, x) \in V \times C \times \mathbf{F}^{n \times n}$. Set

$$
|m|=m_{1}+\cdots+m_{n}
$$

Functions satisfying (2) are obviously homogeneous of degree $2|m|$, so

$$
\rho^{2 m}(G) \subset P_{d}(G), \quad d=2|m| .
$$

The polynomial $\phi_{2 m}$ defined for $x \in G$ by

$$
\phi_{2 m}(x)=|\Delta(x)|^{2 m_{n}} \prod_{j=1}^{n-1}\left|\Delta_{j}(x)\right|^{2\left(m_{j}-m_{j+1}\right)}
$$

is nonzero and easily seen to be in $p^{2 m}(G)$. It is important to note that $\phi_{2 m}$ is nonnegative; in particular, it is positive on the dense open set $V C U$.

2.9 The irreducible representations $\pi_{2 m}$. Clearly, the space $P^{2 m}(G)$ is invariant under right translation by $G$. From the results in $[\mathbf{1 0}]$, it follows that this right action is irreducible and realizes the representation $\pi_{2 m}$ of $G$ with signature $2 m$. Thus, for $a \in G$ and $\phi \in P^{2 m}(G)$

$$
\left(\pi_{2 m}(a) \phi\right)(x)=\phi(x a)
$$

for all $x \in \mathbf{F}^{n \times n}$. In short, the irreducible representation $\pi_{2 m}$ of $G$ with signature $2 m$ is the subrepresentation of $R$ on the subspace $P^{2 m}(G)$.

The function $\phi_{2 m}$ has a group theoretic definition as the highest weight vector of $\pi_{2 m}$. That is to say, $\phi_{2 m}$ is that element of $\rho^{2 m}(G)$, unique up to scalar multiples, for which

$$
\pi_{2 m}(c u) \phi_{2 m}=\mu_{2 m}(c) \phi_{2 m}
$$

for all $(c, u) \in C \times U$. In general, an irreducible representation of $G$ is uniquely determined, up to equivalence, by its highest weight vector.

From the irreducibility of $\pi_{2 m}$, we see that $P^{2 m}(G)$ is the span of the right translates of $\phi_{2 m}$ under the group $G$. That is an alternative, but less explicit, characterization of $p^{2 m}(G)$.

2.10 REMARK. Note that $\pi_{2 m}$ is totally positive, for the evaluation map $e: \phi \rightarrow$ $\phi(1)$ is a linear functional on $P^{2 m}(G)$. Thus, there exists $\phi_{e} \in P^{2 m}(G)$ such that

$$
\phi(1)=\left\langle\phi \mid \phi_{e}\right\rangle
$$

for all $\phi \in \rho^{2 m}(G)$, and the matrix entry $x \rightarrow\left\langle\pi_{2 m}(x) \phi_{2 m} \mid \phi_{e}\right\rangle=\phi_{2 m}(x)$ is nonnegative.

2.11 Inner products. As a subspace of $P(G)$, the representation space $P^{2 m}(G)$ of $\pi_{2 m}$ inherits the inner product (2.2.3). From (2.2.9), the irreducible representation $\pi_{2 m}$ on the space $P^{2 m}(G)$ is unitary when restricted to the maximal compact subgroup $K$. More generally, from (2.2.5)

$$
\pi_{2 m}\left(a^{*}\right)=\left(\pi_{2 m}(a)\right)^{*}
$$

for all $a \in G$. 
3. Invariant polynomials on $G$. The object of this section is to give an explicit description of the decomposition of the space $I(G)=P(G)^{K}$ of left $K$ invariant polynomials on $G$ under the right action of $G$. From remarkable calculations by Hua [18] of character sums, it is known exactly which representations of $G$ appear in $I(G)$; namely, those having signatures of the form $2 m$. Our main contribution is a simple and direct construction of the irreducible subspaces by means of an integral transform, called the spherical transform, on the spaces $p^{2 m}(G)$. Our approach is based on the principle that an irreducible representation appears in $I(G)$ if and only if it is totally positive. Consequently, a representation is totally positive if and only if its signature is of the form $2 \mathrm{~m}$.

3.1 Invariants. Denote by $I(G)$ the subalgebra of $P(G)$ of all left $K$-invariant polynomials; i.e., polynomials $p$ such that $p(k x)=p(x)$ for all $(k, x)$ in $K \times G$. Since $-1 \in K$, there are no nonzero left $K$-invariant polynomials that are homogeneous of an odd degree, so

$$
I(G)=\sum_{d=0}^{\infty} \bigoplus I(G)_{2 d}
$$

where $I(G)_{2 d}$ denotes the subspace of $I(G)$ of polynomials homogeneous of degree $2 d$. Let $\rho$ denote the subrepresentation of $R$ on the subspace $I(G)$ of $\mathcal{P}(G)$. Thus, $\rho$ is the right regular representation of $G$ on $I(G)$. By means of fundamental results of Hua and of Weyl, we describe the decomposition of $\rho$. We state Weyl's theorem [34] on polynomial invariants as a lemma.

3.2 LEMma. Define real-valued polynomials $p_{i}$ for $1 \leq i \leq n$ by

$$
p_{i}(x)=x_{i}^{*} x_{i}
$$

and $p_{i j \varsigma}$ for $1 \leq i<j \leq n$ and $1 \leq \varsigma \leq \nu$ by

$$
p_{i j \zeta}(x)=\left(x_{i}^{*} x_{j}\right)_{\zeta}
$$

when $x_{i}$ is the ith column of $x \in G$. Then the algebra $I(G)$ is generated by the $\omega=n[(n-1) \nu+2] / 2$ polynomials $p_{i}$ and $p_{i j \varsigma}$.

3.3 The spherical transform. For $\phi \in \mathcal{P}(G)$ define the polynomial $\phi^{\#}$ by

$$
\phi^{\#}(x)=\int_{K} \phi(k x) d k
$$

for all $x \in G$. We refer to the map $\Xi: \phi \rightarrow \phi^{\#}$ as the spherical transform for the pair $(G, K)$. It is the orthogonal projection of $P(G)$ onto $I(G)$. Evidently, $\Xi$ commutes with the right action of $G$; that is, $\Xi$ intertwines $R$ with $\rho$.

3.4 THEOREM. Let $d \geq 0$ and $m=\left(m_{1}, \ldots, m_{n}\right)$ with $m_{1} \geq \cdots \geq m_{n} \geq 0$ and $|m|=d$. Then the restriction $\Xi_{2 m}$ of $\Xi$ to $\mathcal{P}^{2 m}(G)$ is an isomorphism of $\bar{P}^{2 m}(G)$ onto a subspace $I^{2 m}(G)$ of $I(G)$, and

$$
I(G)_{2 d}=\sum_{|m|=d} \bigoplus I^{2 m}(G)
$$

is the decompositon of $I(G)_{2 d}$ into irreducible subspaces.

Proof. Clearly, $\Xi$ maps $\rho^{2 m}(G)$ into $I(G)_{2 d}$, and by Schur's lemma $\Xi$ is either injective or else is identically zero. Since $\phi_{2 m} \in p^{2 m}(G)$ is nonnegative, $\Xi_{2 m}$ 
must be injective. Thus, each space $I^{2 m}(G)=\Xi\left(\mathcal{P}^{2 m}(G)\right)$ is an irreducible right invariant subspace of $I(G)$.

From Lemma 3.2, the dimension of the space $I(G)$ is the binomial coefficient

$$
\left(\begin{array}{c}
\omega+d-1 \\
\omega-1
\end{array}\right)
$$

where $\omega=n[(n-1) \nu+2] / 2$. Theorem 3.4 now follows from Hua's formulas $[\mathbf{1 8}$, (1.3.2)-(1.3.5)] which we rewrite as

$$
\left(\begin{array}{c}
\omega+d-1 \\
\omega-1
\end{array}\right)=\sum_{|m|=d} \operatorname{deg} \pi_{2 m} .
$$

3.5 COROLLARY. The representation $\rho$ of $G$ on $I(G)$ is multiplicity free, and an irreducible representation of $G$ appears in $\rho$ if and only if it is totally positive. In particular, an irreducible polynomial representation of $G$ is totally positive if and only if its signature is of the form $2 m$ given by (2.6.1).

PROOF. It suffices to show that a representation which is totally positive must appear in $I(G)_{2 d}$ for some $d$. Therefore, suppose $\pi$ is an irreducible polynomial representation of $G$ on a space $\mathcal{V}$ having a nonnegative matrix entry $f: x \rightarrow$ $\langle\pi(x) u \mid w\rangle$ with $u$ and $w$ nonzero elements of $\mathcal{V}$. Fix $w$ and define the map $A$ from $\mathcal{V}$ into $\mathcal{P}(G)$ by $(A(v))(x)=\langle\pi(x) v \mid w\rangle$ for $v \in \mathcal{V}$ and $x \in G$. Since $\pi$ is irreducible, it acts by scalars on scalar matrices; that is, there exists an integer $l$ such that $\pi(t 1)=t^{l} I$ for all $t \in \mathbf{R}$ where $I$ is the identity on $\mathcal{V}$. Moreover, the function $f=A(u)$ is a nonzero element of $A(\mathcal{V})$. Then $A$ intertwines $\pi$ with the right action of $G$ on the subspace $A(\mathcal{V})$ of $P_{l}(G), A(\mathcal{V}) \neq 0$, and by Schur's Lemma $A$ is injective. Thus, by taking an equivalent representation, if necessary, we may assume that $\pi$ acts by right translation on an irreducible subspace $\mathcal{V}$ of $\mathcal{P}_{l}(G)$ and that there exists a nonzero function in $\mathcal{V}$ that is nonnegative. Now apply the mapping $\Xi$ to $\mathcal{V}$. Then $\Xi(\mathcal{V})$ is a nonzero subspace of $I(G)$ on which the right regular representation of $G$ is equivalent to $\pi$. Of course, $l$ must be even.

3.6 REMARK. The decomposition in the above corollary can be written in symbols as

$$
\rho=\sum_{m} \bigoplus \rho_{2 m}
$$

where

$$
\rho_{2 m}(\cdot)=\Xi_{2 m} \pi_{2 m}(\cdot) \Xi_{2 m}^{-1}
$$

is the irreducible representation of signature $2 m$ acting by right translation on the space $I^{2 m}(G)$.

4. Polynomials on the Hermitian matrices. We translate the preceding decomposition of $I(G)$ into the decomposition of $\mathcal{P}(S)$ under the action $s \rightarrow a^{*} s a$ of $G$, where $S$ denotes the space of Hermitian matrices in $\mathbf{F}^{n \times n}$. In each irreducible subspace there exists a $K$-invariant vector, unique up to constant multiples, called a zonal polynomial. In the language of harmonic analysis, the zonal polynomials are spherical functions for the Gelfand pair $(G, K)$. The essential point is that any $K$-invariant polynomial, or more generally a $K$-invariant analytic function, on $S$ can be expanded in terms of the zonal polynomials. 
4.1 The space $S=S(n, \mathbf{F})$. Denote by $S=S(n, \mathbf{F})$ the real vector space of all $x \in \mathbf{F}^{n \times n}$ such that $x=x^{*}$, on which the group $G$ acts by $s \rightarrow a^{*} s a$ for $s \in S$ and $a \in G$. The cone $P=P(n, \mathbf{F})$ defined in (1.6) is an open subset of $S$. Over the real field, $S$ consists of symmetric matrices; over the complex field, Hermitian matrices, and over the quaternions, quaternionic Hermitian matrices. For convenience, we refer to elements of $S$ as Hermitian matrices, irrespective of the nature of $\mathbf{F}$. The dimension of $S$ over $\mathbf{R}$ is

$$
\omega=n[(n-1) \nu+2] / 2 .
$$

4.2 Polynomials on $S$. Let $P(S)$ denote the algebra of all polynomial functions on $S$, and $P_{d}(S)$ the subspace of polynomials homogeneous of degree $d$. We reserve the symbol $q$ for the generic element of $P(S)$. In analogy to $(2.2 .3)$, we endow $P(S)$ with the inner product

$$
\left\langle q_{1} \mid q_{2}\right\rangle=\left(D\left(\tilde{q}_{2}\right) q_{1}\right)(0)
$$

for $q_{1}$ and $q_{2}$ in $\mathcal{P}(S)$. Define the natural representation $\tau$ of $G$ on $\mathcal{P}(S)$ by

$$
(\tau(a) q)(s)=q\left(a^{*} s a\right)
$$

for $a \in G$ and $q \in \mathcal{P}(S)$. As in (2.2.5)

$$
\left\langle\tau(a) q_{1} \mid \tau\left(a^{\vee}\right) q_{2}\right\rangle=\left\langle q_{1} \mid q_{2}\right\rangle
$$

for all $a \in G$ and $q_{1}, q_{2} \in \mathcal{P}(S)$. In particular, $\tau$ is unitary upon restriction to the maximal compact subgroup $K$.

4.3 The restriction of $\Delta$ to $S$. Recall from (1.2) that $\Delta$ is the determinant on $\mathbf{F}^{n \times n}$ when $\mathbf{F}$ is the real or complex field, but $\Delta$ is the square root of the determinant when $\mathbf{F}$ is the quaternionic division algebra. Nonetheless, the following result shows that the restriction of $\Delta$ to $S$ is a polynomial on $S$, irrespective of the nature of $\mathbf{F}$.

4.4 Proposition. Let $S=S_{n}=S(n, \mathbf{H})$ and $P=P_{n}=P(n, \mathbf{H})$. Then there exists a unique polynomial $\Delta$ on $S$, homogeneous of degree $n$, such that $\Delta(s)^{2}=$ $\operatorname{det} s$ for all $s \in S$ and $\Delta(s)>0$ for $s \in P$.

PROOF. We apply induction on $n$. When $n=1, S_{1}$ is isomorphic to $\mathbf{R}$, and an element of $S_{1}$ has the form $s=\xi 1$ with $\xi \in \mathbf{R}$. In this case, $\Delta(s)=\xi$ and the result is obvious. As induction hypothesis, assume that there exists a polynomial $q=q_{n-1}$ on $S_{n-1}$ such that $q(t)^{2}=\operatorname{det} t$ for all $t \in S_{n-1}$, and such that $q(t)>0$ on $P_{n-1}$. Now suppose $s \in S_{n}$ and set $s_{11}=\xi_{11} 1$ with $\xi_{11} \in \mathbf{R}$. Multiply the first column of $s$ on the left by $\xi_{11}^{-1} s_{1 j}$ and subtract from the $j$ th column $(j=2, \ldots, n)$. Then multiply the first row of the resulting matrix on the left by $\xi_{11}^{-1} s_{1 j}^{*}$ and subtract from the $j$ th row $(j=2, \ldots, n)$. This yields

$$
u=e^{*} s e=\left(\begin{array}{cc}
\xi_{11} 1 & 0 \\
0 & t
\end{array}\right)
$$

with $\operatorname{det} e=1$ and $t \in S_{n-1}$ given by

$$
t_{i j}=s_{i j}-\xi_{11}^{-1} s_{1 j} s_{1 i}^{*}, \quad i \leq j .
$$

Clearly, $t \in P_{n-1}$ when $s \in P_{n}$. By the induction hypothesis

$$
\operatorname{det} s=\operatorname{det} u=\xi_{11}^{2} q(t)^{2}=\xi_{11}^{-2(n-2)} q\left(\xi_{11} s_{i j}-s_{1 j} s_{1 i}^{*}\right)^{2}
$$


for all $s \in S_{n}$. Note that $\xi_{11}^{n-2}$ divides the polynomial $s \rightarrow q\left(\xi_{11} s_{i j}-s_{1 j} s_{1 i}^{*}\right)$. Thus,

$$
\Delta(s)=q_{n}(s)=\xi_{11}^{-(n-2)} q_{n-1}\left(\xi_{11} s_{i j}-s_{1 j} s_{1 i}^{*}\right)
$$

defines a polynomial $\Delta$ on $S, \operatorname{det} s=\Delta(s)^{2}$, and $\Delta(s)>0$ for all $s \in P_{n}$. This completes the proof by induction.

4.5 REMARKS. From (1.7), recall polar coordinates $x=k r^{1 / 2}$ on $G$. Since $P$ is open in $S$, any polynomial on $S$ is uniquely determined by its restriction to $P$; and conversely, any polynomial on $P$ extends uniquely to a polynomial on $S$. We observe that the dimension (4.1.1) of $S$ is the same number $\omega$ that appears in Lemma 3.2. Indeed, if $x_{i}$ denotes the $i$ th column of $x$, then the $i j$ th entry of $r=x^{*} x$ is $r_{i j}=x_{i}^{*} x_{j}$, and (3.2.1) and (3.2.2) can be rewritten as

$$
p_{i}(x)=q_{i}(r)=r_{i i} \quad \text { and } \quad p_{i j \zeta}(x)=q_{i j \zeta}(r)=\left(r_{i j}\right)_{\varsigma} .
$$

More generally, for any polynomial $p$ in $I(G)$ the formula

$$
q\left(x^{*} x\right)=p(x)
$$

is well defined and yields a polynomial $q$ on $S$. Thus, the map

$$
\Omega: p \rightarrow q
$$

takes $I(G)$ isomorphically onto $P(S)$, and intertwines the representations $\rho$ and $\tau$. That is,

$$
\Omega \rho(a)=\tau(a) \Omega
$$

for all $a \in G$. Clearly, for any $d, \Omega$ takes $I_{2 d}(G)$ to $P_{d}(S)$. By means of the isomorphism $\Omega$ we can restate the decomposition of $I(G)$ in terms of polynomials on $S$.

4.6 THEOREM. Fix $d \geq 0$ and let $m$ be as in Theorem 3.4. Denote by $\Omega_{2 m}$ the restriction of $\Omega$ to $I^{2 m}(G)$, and set

$$
\rho^{m}(S)=\Omega_{2 m}\left(I^{2 m}(G)\right) \quad \text { and } \quad \tau_{2 m}(\cdot)=\Omega_{2 m} \rho_{2 m}(\cdot) \Omega_{2 m}^{-1}
$$

where $\rho_{2 m}$ is given by (3.6.2). Then $\tau_{2 m}$ is the irreducible representation of $G$ with signature $2 m$ acting in the subspace $P^{m}(S)$ of $P_{d}(S) ; P_{d}(S)$ decomposes as

$$
P_{d}(S)=\sum_{|m|=d} \bigoplus^{p^{m}}(S)
$$

and the representation $\tau$ of $G$ on $\mathcal{P}(S)$ is multiplicity free with decomposition

$$
\tau=\sum_{m} \bigoplus \tau_{2 m}
$$

4.7 Highest weight vectors. Let $|m|=d$ and set

$$
q_{m}(s)=\Delta(s)^{m_{n}} \prod_{j=1}^{n-1} \Delta_{j}(s)^{m_{j}-m_{j+1}}
$$

for $s \in S, \Delta_{j}$ being given by (1.4.3). Then $q_{m}$ is a homogeneous polynomial of degree $d$, and

$$
\tau(c u) q_{m}=\mu_{2 m}(c) q_{m}
$$

for all $(c, u) \in C \times U$, where $\mu_{2 m}$ is the character of $C$ given by (2.8.1). It follows from (2.9) that $q_{m}$ is in $P^{m}(S)$ and $q_{m}$ is the highest weight vector of $\tau_{2 m}$. 
4.8 THEOREM. In each irreducible subspace $\mathrm{P}^{m}(S)$ of $\mathcal{P}(S)$ there is a nonzero $K$-invariant function $f_{m}$ which is unique up to constant multiples. That is, there exists a nonzero function $f_{m}$ in $P^{m}(S)$, unique up to constant multiples, such that

$$
f_{m}\left(k^{*} s k\right)=f_{m}(s)
$$

for all $s \in S$ and $k \in K$.

ProOF. There is certainly some nonzero $K$-invariant polynomial in $p^{m}(S)$. For example, the polynomial $f_{m}$ defined for $s \in S$ by

$$
f_{m}(s)=\int_{K} q_{m}\left(k^{*} s k\right) d k
$$

is in $P^{m}(S)$, is $K$-invariant, and is positive on the positive cone $P$. That such a polynomial is unique can be seen directly as follows. Fix $d \geq 0$ and suppose $|m|=d$. Since any Hermitian matrix $s$ can be diagonalized by a matrix in $K$, it follows from (1) that $f_{m}$ is uniquely determined by its restriction to the diagonal matrices in $S$. Thus, as a polynomial in the $n$ (real) diagonal entries which is homogeneous of degree $d, f_{m}$ is invariant under the action of the symmetric group on $n$ letters. The dimension of the space of such polynomials is precisely the number of partitions $m$ of the positive integer $d$, which shows that there can be at most one linearly independent $K$-invariant element in $p^{m}(S)$.

4.9 Zonal polynomials. A nonzero $K$-invariant polynomial in $p^{m}(S)$ is called a zonal polynomial on $S$ of weight $m$. The collection of all zonal polynomials, constant multiples identified, is in one-to-one correspondence with the collection of irreducible representations of $G$ which are totally positive. Clearly, the algebra $P(S)^{K}$ of all $K$-invariant polynomials on $S$ is spanned by the zonal polynomials; that is,

$$
P(S)^{K}=\sum_{m} \bigoplus \mathbf{C} f_{m}
$$

In particular, zonal polynomials corresponding to different signatures are orthogonal.

4.10 Complexification and compact forms. There is an alternative representationtheoretic proof of Theorem 4.8 that illustrates the connection between the noncompact space $K \backslash G$ and its compact counterpart. Let $G^{\mathbf{C}}$ be the complexification of $G, K^{\mathbf{C}}$ the complexification of $K$ in $G^{\mathbf{C}}$, and $L$ the maximal compact subgroup (compact real form) of $G^{\mathbf{C}}$. The group $G^{\mathbf{C}}$ is the collection of all invertible elements of the complexification of the algebra $\mathbf{F}^{n \times n}$. The specific structures are given below.

\begin{tabular}{cccccc}
\hline $\mathbf{F}$ & $\mathbf{F}^{\mathbf{C}}$ & $G^{\mathbf{C}}$ & $L$ & $K \backslash L$ & $K \backslash G$ \\
\hline $\mathbf{R}$ & $\mathbf{C}$ & $G L(n, \mathbf{C})$ & $\mathcal{L}(n)$ & $O(n) \backslash U(n)$ & $O(n) \backslash G L(n, \mathbf{R})$ \\
$\mathbf{C}$ & $\mathbf{C} \oplus \mathbf{C}$ & $G L(n, \mathbf{C}) \times G L(n, \mathbf{C})$ & $\mathcal{U}(n) \times \mathcal{U}(n)$ & $\operatorname{diag} \mathcal{U}(n) \backslash U(n) \times U(n)$ & $\mathcal{U}(n) \backslash G L(n, \mathbf{C})$ \\
$\mathbf{H}$ & $\mathbf{C}^{2 \times 2}$ & $G L(2 n, \mathbf{C})$ & $U(2 n)$ & $S p(n) \backslash U(2 n)$ & $S p(n) \backslash G L(n, \mathbf{H})$ \\
\hline
\end{tabular}

The chain of mappings

$$
\rho^{m}(S) \rightarrow I^{2 m}(G) \rightarrow I^{2 m}\left(G^{\mathbf{C}}\right) \rightarrow \rho^{m}(K \backslash L)
$$


that results from complexification of a $K$-invariant polynomial on $G$ to a $K^{\mathrm{C}}$ invariant polynomial on $G^{\mathbf{C}}$ and then restriction of that polynomial to $K \backslash L$, provides an isomorphism of $P^{m}(S)$ with a subspace $P^{m}(K \backslash L)$ of continuous functions on $K \backslash L$ that is invariant and irreducible for the action of $L$. Then the decomposition

$$
P(S)=\sum_{m} \bigoplus^{P^{m}}(S)
$$

of the space of polynomials on $S$ corresponds to the multiplicity free decomposition

$$
L^{2}(K \backslash L)=\sum_{m} \bigoplus^{\rho^{m}}(K \backslash L)
$$

of the square-integrable functions on the compact form $K \backslash L$ of $K \backslash G$. The fact that each space $P^{m}(K \backslash L)$ has an essentially unique $K$-invariant function is a consequence of Frobenius reciprocity for compact groups [7]. Theorem 4.8 then follows by lifting this result from $P^{m}(K \backslash L)$ to $P^{m}(S)$ via the above maps. In this context, the multiplicity free nature of $P(S)$ is a reflection of the fact that $K \backslash L$ is a compact Riemannian symmetric space $[\mathbf{1 3}]$. Note that in the complex case, $K \backslash L$ is the symmetric space for the compact group $U(n)$, and (3) is precisely the Peter-Weyl theorem for $L^{2}(U(n))$. This has the following obvious consequence.

4.11 Proposition. Let $\mathbf{F}=\mathbf{C}$ and $\lambda_{m}=\pi_{m \times 0}$ be the holomorphic representation of $G L(n, \mathbf{C})$ with signature $m \times 0(c f .(2.4 .2))$. Then the zonal polynomial $f_{m}$ is given by the formula

$$
f_{m}(s)=\left(\operatorname{deg} \lambda_{m}\right)^{-1} \operatorname{tr}\left(\lambda_{m}(s)\right)
$$

for all $s \in S$.

In other words, in the complex case the zonal polynomials are the restriction to $S$ of the (normalized) irreducible holomorphic characters of $G L(n, \mathbf{C})$.

5. Properties of zonal polynomials. In this section we derive a number of properties of the zonal polynomials. We do not develop the theory in fine detail, but rather focus on those results necessary for our treatment of hypergeometric functions on $S$. In particular, we normalize the zonal polynomial $Z_{m}$ of weight $m$ in such a way as to reduce to unity all the coefficients in the expansion of the powers of the trace. We also derive a Laplace transform formula and an Euler-type integral for the zonal polynomials, the latter being in the spirit of [6] and [19]. Our approach highlights the significance for analysis on $S$ of a generalization of the classical gamma function. This generalized gamma function is scalar-valued, but it is intimately related to an operator-valued version, defined previously in [9] in connection with Fourier analysis on $\mathbf{F}^{n \times n}$.

5.1 Normalization. The zonal polynomial $f_{m}$ of weight $m$ defined by $(4.8 .2)$ is specified by the normalization

$$
f_{m}(1)=1
$$

However, a more convenient normalization relates the zonal polynomials of weight $m$ to the $K$-invariant polynomial $s \rightarrow(\operatorname{tr} s)^{d}$ where $|m|=d$. 
5.2 LemMA. For any $d \geq 0$

$$
(\operatorname{tr} s)^{d}=\sum_{|m|=d} \alpha_{m} f_{m}(s)
$$

where $\alpha_{m}=(d !)\left\|f_{m}\right\|^{-2}>0$ for all $m$.

PROOF. Set $e_{t}(s)=\exp (\operatorname{tr} s t)$ for $s, t \in S$. By straightforward differentiation,

$$
\left(D\left(f_{m}\right) e_{t}\right)(s)=f_{m}(t) e_{t}(s)
$$

and hence

$$
\left(D\left(f_{m}\right) e_{1}\right)(0)=f_{m}(1)=1 .
$$

Now, since $e_{1}$ is $K$-invariant, it has an expansion of the form

$$
e_{1}=\sum_{m} \beta_{m} f_{m}
$$

with coefficients $\beta_{m}$ that are determined by substitution into (3). Thus, by (4.2.1)

$$
\sum_{m^{\prime}} \beta_{m^{\prime}}\left(D\left(f_{m}\right) f_{m^{\prime}}\right)(0)=\sum_{m^{\prime}} \beta_{m^{\prime}}\left\langle f_{m^{\prime}} \mid f_{m}\right\rangle=1
$$

and since the polynomials $f_{m}$ and $f_{m^{\prime}}$ are orthogonal it follows that $\beta_{m}=\left\|f_{m}\right\|^{-2}$ $>0$. Finally, evaluate both sides of (4) at $\xi s$, where $\xi \in \mathbf{R}$ and $s \in S$, to obtain

$$
\sum_{d=0}^{\infty} \frac{\xi^{d}(\operatorname{tr} s)^{d}}{d !}=\sum_{d=0}^{\infty} \xi^{d}\left(\sum_{|m|=d} \beta_{m} f_{m}(s)\right)
$$

from which the lemma follows by equating like coefficients of $\xi^{d}$.

5.3 Definition. We define the normalized zonal polynomial $Z_{m}$ of weight $m$ by

$$
Z_{m}(s)=\left(d ! /\left\|f_{m}\right\|^{2}\right) f_{m}(s)
$$

where $d=|m|$, in terms of which

$$
(\operatorname{tr} s)^{d}=\sum_{|m|=d} Z_{m}(s)
$$

for all $s \in S$. In particular, (1) implies that

$$
Z_{m}(1)=d ! /\left\|f_{m}\right\|^{2}>0 .
$$

This formula for $Z_{m}(1)$ is sufficient for our needs. However, the value of $Z_{m}(1)$ has been computed explicitly in both the real and complex cases [19]. For the quaternionic case, there appears to be no literature on $Z_{m}(1)$; however, $Z_{m}(1)$ will be computed from techniques to appear in a future paper [12]. We remark that in the statistical literature $[\mathbf{1 9}, \mathbf{2 3}]$ the symbol $C_{m}$ is used, rather than $Z_{m}$, for the zonal polynomial normalized by $(2)$.

5.4 REMARKS. Let $Z=Z_{m}$. Recall from (4.5) that $\Omega$ is an isomorphism of $I(G)$ with $\mathcal{P}(S)$. Denote by $p_{Z}$ the inverse image of $Z$ under $\Omega$; that is,

$$
Z\left(x^{*} x\right)=p_{Z}(x)
$$


for all $x \in G$. Then $p_{Z}$ spans the unique one-dimensional subspace of $I(G)^{2 m}$ consisting of right $K$-invariant polynomials. As all functions in $I(G)^{2 m}$ are left $K$-invariant,

$$
p_{Z}\left(k_{1} x k_{2}\right)=p_{Z}(x)
$$

for all $k_{1}, k_{2} \in K$ and $x \in G$. Any $x \in G$ can be decomposed as $x=k_{1} c k_{2}$ with $k_{1}, k_{2} \in K$ and $c \in C^{+}$, where $C^{+}$is the subgroup defined in (2.1) of positive diagonal matrices. Hence, the polynomial $p_{Z}$ on $G$ is uniquely determined by restriction to the positive diagonal matrices. Consequently,

$$
p_{Z}\left(x^{*}\right)=p_{Z}(x)
$$

for all $x \in G$, and

$$
p_{Z}\left(x y^{*}\right)=p_{Z}\left(y x^{*}\right)
$$

for all $x, y \in G$. Rewriting $p_{Z}\left(x y^{*}\right)$ as $\left(\rho_{2 m}\left(y^{*}\right) p_{Z}\right)(x)$, we see that $p_{Z}\left(x y^{*}\right)$ lies in $I(G)^{2 m}$, as a function of either $x$ or $y$. By means of (1), the above properties of $p_{Z}$ translate into the following properties of $Z$.

The value of $Z$ at $s \in S$ is uniquely determined by the eigenvalues of $s$; that is,

$$
Z(s)=Z(\lambda)
$$

where $\lambda$ is a diagonal matrix, the diagonal entries of which are the eigenvalues of $s$. If we set $s=x^{*} x$ and $t=y^{*} y$, then (4) becomes

$$
Z\left(y s y^{*}\right)=Z\left(x t x^{*}\right)
$$

and from polar coordinates (1.7) and the $K$-invariance of $Z$

$$
Z\left(t^{1 / 2} s t^{1 / 2}\right)=Z\left(s^{1 / 2} t s^{1 / 2}\right) .
$$

The formula in (7), as a function of $s$ and $t$, extends uniquely from $P \times P$ to a polynomial on $S \times S$ which lies in $P^{m}(S)$ as a function of either $s$ or $t$. Moreover, the matrices $s t, t s, s^{1 / 2} t s^{1 / 2}$, and $t^{1 / 2} s t^{1 / 2}$ all have the same eigenvalues. Thus, we opt for convenience of notation rather than strict adherence to rigor, and write $Z(s t)$ or $Z(t s)$ in place of $Z\left(t^{1 / 2} s t^{1 / 2}\right)$, even though $s t$ or $t s$ need not lie in $S$. Note that

$$
Z_{m}\left(r^{1 / 2} s r^{1 / 2} t\right)=Z_{m}\left(s r^{1 / 2} t r^{1 / 2}\right)
$$

for all $s, t \in S$ and $r \in P$. The following functional equation for zonal polynomials is a mean-value theorem that, in the general theory [14], is characteristic of spherical functions.

5.5 Proposition. For any $s, t \in S$,

$$
\int_{K} Z_{m}\left(s k^{-1} t k\right) d k=\frac{Z_{m}(s) Z_{m}(t)}{Z_{m}(1)}
$$

where $d k$ is normalized Haar measure on $K$.

Proof. Set $Z=Z_{m}$ and $p=p_{Z}$ and let $s=x^{*} x$ and $t=y^{*} y$ for $x, y \in G$. By (5.4), it is sufficient to prove that

$$
\int_{K} p\left(y k x^{*}\right) d k=\frac{p(x) p(y)}{p(1)} .
$$


The left side of (2) defines a polynomial on $G \times G$ which, as a function of $y$, is a right $K$-invariant element of $I(G)^{2 m}$. Hence, there exists a polynomial $\psi$ on $G$ such that the left side of $(2)$ is equal to $\psi(x) p(y)$. As a function of $x$, the left side of (2) is again a right $K$-invariant element of $I(G)^{2 m}$, from which we conclude that $\psi(x)=\alpha p(x)$ for some constant $\alpha$. However, when $x=y=1$ the left side of (2) is equal to $p(1)$, from which it follows that $\alpha=1 / p(1)$.

5.6 The gamma function for $P$. Let $q_{m} \in P^{m}(S)$ be the highest weight vector (4.7.1) for $\tau_{2 m}$, and let $\alpha$ be a complex variable. Set

$$
\Gamma_{n}(\alpha, m, \mathbf{F})=\Gamma_{n}(\alpha, m)=\int_{P} e^{-\operatorname{tr} r} \Delta(r)^{\alpha} q_{m}(r) d_{*} r
$$

whenever the integral converges. We refer to the function $\Gamma$ as the generalized gamma function for the cone $P=P(n, \mathbf{F})$. For fixed $m=\left(m_{1}, \ldots, m_{n}\right)$, the function $\alpha \rightarrow \Gamma_{n}(\alpha, m)$ is said to be the gamma function of weight $m$ for the cone $P$. When $m=(0, \ldots, 0)$, which corresponds to the identity representation of $G$, we drop the dependence upon $m$ and set

$$
\Gamma_{n}(\alpha, \mathbf{F})=\Gamma_{n}(\alpha)=\int_{P} e^{-\operatorname{tr} r} \Delta(r)^{\alpha} d_{*} r .
$$

Define the generalized Pochhammer symbol of weight $m$ by

$$
[\alpha ; \mathbf{F}]_{m}=[\alpha]_{m}=\prod_{j=1}^{n}\left(\alpha-\frac{1}{2}(j-1) \nu\right)_{m_{j}}
$$

where

$$
(\alpha)_{j}=\alpha(\alpha+1) \cdots(\alpha+j-1)
$$

is the standard Pochhammer symbol.

5.7 THEOREM. The integral (5.6.1) defining $\Gamma_{n}(\alpha, m)$ converges if and only if

$$
\operatorname{Re} \alpha>\frac{1}{2}(n-1) \nu-m_{n} \text {. }
$$

In this half-plane

$$
\Gamma_{n}(\alpha, m)=\pi^{n(n-1) \nu / 4} \prod_{j=1}^{n} \Gamma\left(\alpha+m_{j}-\frac{1}{2}(j-1) \nu\right)
$$

where $\Gamma$ is the ordinary gamma function, and for any $m$ the mapping $\alpha \rightarrow \Gamma_{n}(\alpha, m)^{-1}$ extends to an entire function of $\alpha$. In particular,

$$
\Gamma_{n}(\alpha)=\pi^{n(n-1) \nu / 4} \prod_{j=1}^{n} \Gamma\left(\alpha-\frac{1}{2}(j-1) \nu\right)
$$

and

$$
\Gamma_{n}(\alpha, m)=[\alpha]_{m} \Gamma_{n}(\alpha)
$$

ProOF. From (1.6.2) and (4.7.2)

$$
\begin{aligned}
\Gamma_{n}(\alpha, m) & =2^{n} \int_{U} \int_{C^{+}} e^{-\operatorname{tr}\left(c u^{*} u c\right)} \Delta(c)^{2 \alpha} q_{m}\left(c u^{*} u c\right) d u d_{*} c \\
& =2^{n} \int_{U} \int_{C^{+}} e^{-\operatorname{tr}\left(c u^{*} u c\right)} \Delta(c)^{2 \alpha}\left(\tau_{2 m}(u c) q_{m}\right)(1) d u d_{*} c \\
& =2^{n} \int_{U} \int_{C^{+}} e^{-\operatorname{tr}\left(c u^{*} u c\right)} \Delta(c)^{2 \alpha} \mu_{2 m}(c) d u d_{*} c .
\end{aligned}
$$


Let $W$ denote the vector space of all upper triangular nilpotent $n \times n$ matrices $w$ over $\mathbf{F}$, and let $u=1+w$ for any $u \in U$. Since $w$ is nilpotent and $c$ is diagonal,

$$
\begin{aligned}
\Gamma_{n}(\alpha, m) & =2^{n} \int_{C^{+}}\left(\int_{W} e^{-\operatorname{tr}\left(c w^{*} w c\right)} d w\right) e^{-\operatorname{tr}\left(c^{2}\right)} \Delta(c)^{2 \alpha} \mu_{2 m}(c) d_{*} c \\
& =2^{n} \pi^{n(n-1) \nu / 4} \int_{C^{+}} e^{-\operatorname{tr}\left(c^{2}\right)} \Delta(c)^{2 \alpha} \mu_{2 m}(c) J\left(c^{-1}\right) d_{*} c
\end{aligned}
$$

where $J(c)=\left(c_{2} c_{3}^{2} \cdots c_{n}^{n-1}\right)^{\nu}$ is the Jacobian of the transformation $w \rightarrow w c$. From (1.6.1)

$$
\Gamma_{n}(\alpha, m)=2^{n} \pi^{n(n-1) \nu / 4} \prod_{j=1}^{n} \int_{0}^{\infty}\left\{\exp \left(-c_{j}^{2}\right)\right\} c_{j}^{2 \alpha+2 m_{j}-(j-1) \nu} c_{j}^{-1} d c_{j}
$$

which is finite if and only if $\alpha$ lies in the half-plane defined by (1), and in this domain

$$
\Gamma_{n}(\alpha, m)=\pi^{n(n-1) \nu / 4} \prod_{j=1}^{n} \Gamma\left(\alpha+m_{j}-\frac{1}{2}(j-1) \nu\right) .
$$

Formula (3) is the special case in which $m=(0, \ldots, 0)$, and (4) follows by means of the functional equation for the ordinary gamma function.

5.8 REMARKS. (1) From (5.6.2) and (5.7.3), it is now straightforward to verify formula (1.7.2) for the constant $\beta_{n}$ that enters integration over $G$ in polar coordinates. One integrates the function

$$
x \rightarrow|\Delta(x)|^{\nu n / 2} e^{-\operatorname{tr}\left(x^{*} x\right)} .
$$

The left side of (1.7.2) becomes $\pi^{\nu n^{2} / 2}$, and the right side becomes

$$
\beta_{n} \pi^{n(n-1) \nu / 4} \prod_{j=1}^{n} \Gamma\left(\frac{(n-j+1) \nu}{2}\right) .
$$

(2) The calculation of the integral (5.6.1) is implicit in $\S 4$ of [9] in connection with the absolute convergence of a certain operator-valued gamma function that relates to analysis of vector-valued functions on $\mathbf{F}^{n \times n}$.

(3) We close this section by deriving a Laplace transform formula and an Eulertype integral for the zonal polynomials. We utilize the complexification $S^{\mathbf{C}}=S+i S$ of $S$. That is, $S^{\mathbf{C}}$ consists of all matrices $x \in\left(\mathbf{F}^{\mathbf{C}}\right)^{n \times n}$ of the form $z=x+i y$ with $x, y \in S$. We refer to $x=\operatorname{Re} z$ and $y=\operatorname{Im} z$ as the real and imaginary parts of $z$, respectively. The (generalized) right half-plane $\Phi=P+i S$ in $S^{\mathbf{C}}$ consists of all $z \in S^{\mathbf{C}}$ such that $\operatorname{Re} z \in P$.

5.9 THEOREM. Let $\alpha$ and $m$ satisfy (5.7.1). Then

$$
\int_{P} e^{-\operatorname{tr}(r z)} \Delta(r)^{\alpha} Z_{m}(r s) d_{*} r=[\alpha]_{m} \Gamma_{n}(\alpha) \Delta(z)^{-\alpha} Z_{m}\left(s z^{-1}\right)
$$

for all $s \in S$ and $z$ in the generalized right half-plane $\Phi$. 
Proof. Denote the left side of (1) by $I(s, z)$. By (4.8.2), (5.3), an interchange of order of integration, and (5.7.4)

$$
\begin{aligned}
I(1,1) & =\int_{P} e^{-\operatorname{tr} r} \Delta(r)^{\alpha} Z_{m}(r) d_{*} r \\
& =Z_{m}(1) \int_{P} e^{-\operatorname{tr} r} \Delta(r)^{\alpha}\left(\int_{K} q_{m}\left(k^{-1} r k\right) d k\right) d_{*} r \\
& =Z_{m}(1) \int_{P} e^{-\operatorname{tr} r} \Delta(r)^{\alpha} q_{m}(r) d_{*} r \\
& =Z_{m}(1) \Gamma_{n}(\alpha, m)=Z_{m}(1)[\alpha]_{m} \Gamma_{n}(\alpha)
\end{aligned}
$$

Next, since the function $s \rightarrow I(s, 1)$ is $K$-invariant and in $\mathcal{P}^{m}(S)$, there exists a constant $c$ such that $I(s, 1)=c Z_{m}(s), s \in S$. Clearly, $c=I(1,1) / Z_{m}(1)$ so

$$
I(s, 1)=[\alpha]_{m} \Gamma_{n}(\alpha) Z_{m}(s) .
$$

Now, let $z \in P$ and make the change of variable $r \rightarrow z^{-1 / 2} r z^{-1 / 2}$ in the integral defining $I(s, z)$. Then by $(5.4 .8)$

$$
I(s, z)=\Delta(z)^{-\alpha} I\left(z^{-1 / 2} s z^{-1 / 2}, 1\right)
$$

and hence

$$
I(s, z)=[\alpha]_{m} \Gamma_{n}(\alpha) \Delta(z)^{-\alpha} Z_{m}\left(z^{-1 / 2} s z^{-1 / 2}\right) .
$$

Thus, for $z \in P$ and $s \in S$

$$
I(s, z)=[\alpha]_{m} \Gamma_{n}(\alpha) \Delta(z)^{-\alpha} Z_{m}\left(s z^{-1}\right) .
$$

The result now follows by analytic continuation in $z$ from $P$ to $\Phi=P+i S$.

5.10 CoROllaRY. Let $\operatorname{Re}(\alpha)>(n-1) \nu / 2$ and $\operatorname{Re}(\beta-\alpha)>(n-1) \nu / 2$. Then

$$
\int_{0<r<1} \Delta(r)^{\alpha} \Delta(1-r)^{\beta-\alpha-\theta} Z_{m}(r z) d_{*} r=\frac{\Gamma_{n}(\alpha) \Gamma_{n}(\beta-\alpha)}{\Gamma_{n}(\beta)} \frac{[\alpha]_{m}}{[\beta]_{m}} Z_{m}(z)
$$

for all $z \in S^{\mathrm{C}}=S+i S$, where $\theta$ is given by (1.6.4).

PROOF. From our proof of Theorem 5.7, $\int_{0<r<1} \Delta(r)^{\alpha} d_{*} r<\infty$ if and only if $\operatorname{Re} \alpha>(n-1) \nu / 2$. Therefore, the conditions on $\alpha$ and $\beta$ in the statement of the corollary are precisely those for which the integral in (1) converges absolutely.

Denote the integral in (1) by $I(z)$. For the moment, let $z \in S$. From the $K$ invariance of the measure $d_{*} r$ on the set $P_{1}$ of all $0<r<1$, it follows that $I$ is a $K$-invariant polynomial which is clearly in $\mathcal{P}^{m}(S)$. Hence, $I$ is a constant multiple of $Z_{m}$; explicitly,

$$
I(z)=I(1) Z_{m}(z) / Z_{m}(1)
$$

for all $z \in S$. By analytic continuation, (2) is valid for all $z \in S^{\mathbf{C}}$. Therefore, it remains to compute $I(1)$. We proceed as follows. By Theorem 5.9 and (2),

$$
\begin{aligned}
\Gamma_{n}(\beta)[\beta]_{m} I(1) & =\left(\int_{P} e^{-\operatorname{tr}(r)} \Delta(r)^{\beta} Z_{m}(r) d_{*} r\right) \frac{I(1)}{Z_{m}(1)} \\
& =\int_{P} e^{-\operatorname{tr}(r)} \Delta(r)^{\beta} I(r) d_{*} r
\end{aligned}
$$


Substituting for $I(r)$ in this last integral, we obtain

$$
[\beta]_{m} \Gamma_{n}(\beta) I(1)=\int_{P}\left(\int_{P_{1}} e^{-\operatorname{tr}(r)} \Delta(r)^{\beta} \Delta(t)^{\alpha}(1-t)^{\beta-\alpha-\theta} Z_{m}(\operatorname{tr}) d_{*} t\right) d_{*} r .
$$

Next, make the substitution $t \rightarrow r^{-1 / 2} \operatorname{tr}^{-1 / 2}$ in one integral, followed by $r \rightarrow r+t$ in the other. Then

$$
\begin{aligned}
{[\beta]_{m} \Gamma_{n}(\beta) I(1) } & =\int_{P} \int_{P} e^{-\operatorname{tr}(r+t)} \Delta(t)^{\alpha} \Delta(r)^{\beta-\alpha} Z_{m}(t) d_{*} t d_{*} r \\
& =\left(\int_{P} e^{-\operatorname{tr}(r)} \Delta(r)^{\beta-\alpha} d_{*} r\right)\left(\int_{P} e^{-\operatorname{tr}(t)} \Delta(t)^{\alpha} Z_{m}(t) d_{*} t\right) \\
& =[\alpha]_{m} \Gamma_{n}(\alpha) \Gamma_{n}(\beta-\alpha) Z_{m}(1),
\end{aligned}
$$

which yields the desired result.

6. Hypergeometric functions on $S$. In the previous sections we have assembled all the machinery required to introduce hypergeometric series on $S$. These series are natural generalizations of the classical hypergeometric series, and are appropriate to analysis on $S$. Over the real field, the generalized hypergeometric functions of matrix argument were defined by Herz [15], and more generally for certain symmetric spaces by Gindikin [6]. The former adopts the approach in which the hypergeometric functions are defined inductively by means of the Laplace transform and inverse Laplace transform, while the latter generalizes the Gaussian ${ }_{2} F_{1}$ by means of Euler integrals. Neither develops series expansions. Our approach here is more direct. In analogy to the familiar classical case in which hypergeometric functions are given by power series expansions, we define (generalized) hypergeometric functions on $S$ as infinite series of zonal polynomials, and subsequently we prove Laplace transform formulas and Euler-type integrals to make the connection with the earlier methods. Over the real field, this approach was pioneered by the statisticians James [19] and Constantine [2]. Although our methods differ, our results reduce to theirs for the real field. However, our proof of convergence in Theorem 6.3 fills an apparent gap in the statistics literature.

Throughout, we let $\|s\|=\max \left\{\left|\lambda_{i}\right|: i=1, \ldots, n\right\}$ where $\lambda_{1}, \ldots, \lambda_{n}$ are the eigenvalues of $s \in S$.

6.1 Definition. Let $\alpha_{1}, \ldots, \alpha_{p}, \beta_{1}, \ldots, \beta_{q} \in \mathbf{C}$ such that $-\beta_{i}+(j-1) \nu / 2$ $(1 \leq i \leq q, 1 \leq j \leq n)$ is not a nonnegative integer. The hypergeometric function with argument $s \in S$ and parameters $\alpha_{1}, \ldots, \alpha_{p}$ and $\beta_{1}, \ldots, \beta_{q}$ is defined by the series

$$
{ }_{p} F_{q}\left(\alpha_{1}, \ldots, \alpha_{p} ; \beta_{1}, \ldots, \beta_{q} ; s\right)=\sum_{d=0}^{\infty} \sum_{|m|=d} \frac{\left[\alpha_{1}\right]_{m} \cdots\left[\alpha_{p}\right]_{m}}{\left[\beta_{1}\right]_{m} \cdots\left[\beta_{q}\right]_{m}} \frac{Z_{m}(s)}{d !}
$$

6.2 EXAMPLES. First, from (5.3.2)

$$
{ }_{0} F_{0}(s)=\sum_{d=0}^{\infty} \sum_{|m|=d} \frac{Z_{m}(s)}{d !}=\sum_{d=0}^{\infty} \frac{(\operatorname{tr} s)^{d}}{d !}=e^{\operatorname{tr} s}
$$

Note that the hypergeometric series ${ }_{0} F_{0}$ converges absolutely for all $s \in S$; indeed, for all $s$ in the complexification $S^{\mathbf{C}}$ of $S$. This is characteristic of the general 
situation when $p \leq q$. Next, we sum the hypergeometric series ${ }_{1} F_{0}$. If $\operatorname{Re} \alpha>$ $(n-1) \nu / 2$ and $\|s\|<1$, then by Theorem 5.9 and Fubini's theorem

$$
\begin{aligned}
\Delta(1-s)^{-\alpha} & =\frac{1}{\Gamma_{n}(\alpha)} \int_{P} e^{-\operatorname{tr}((1-s) r) \Delta(r)^{\alpha} d_{*} r} \\
& =\frac{1}{\Gamma_{n}(\alpha)} \int_{P} e^{-\operatorname{tr} r} F_{0}(r s) \Delta(r)^{\alpha} d_{*} r \\
& =\frac{1}{\Gamma_{n}(\alpha)} \sum_{d=0}^{\infty} \sum_{|m|=d} \frac{1}{d !} \int_{P} e^{-\operatorname{tr} r} \Delta(r)^{\alpha} Z_{m}(r s) d_{*} r \\
& =\sum_{d=0}^{\infty} \sum_{|m|=d} \frac{[\alpha]_{m} Z_{m}(s)}{d !}={ }_{1} F_{0}(\alpha ; s) .
\end{aligned}
$$

Thus,

$$
{ }_{1} F_{0}(\alpha ; s)=\Delta(1-s)^{-\alpha}
$$

gives the full analytic continuation of ${ }_{1} F_{0}(\alpha ; \cdot)$ to any simply-connected domain in $S^{\text {C }}$. The right side of $(2)$ is determined by the principal branch of the argument. The fact that the hypergeometric series ${ }_{1} F_{0}$ has $\{s \in S:\|s\|<1\}$ as its domain of convergence is characteristic of ${ }_{p+1} F_{p}$ for all $p \geq 0$.

6.3 THEOREM (CONVERGENCE OF HYPERGEOMETRIC FUNCTIONS). (1) If $p \leq q$ then the hypergeometric series (6.1.1) converges absolutely for all $s \in S$.

(2) If $p=q+1$ then the series (6.1.1) converges absolutely for $\|s\|<1$ and diverges for $\|s\|>1$.

(3) If $p>q$ then the series (6.1.1) diverges unless it terminates.

6.4 Outline of proof. The strategy is to reduce the proof of convergence of (6.1.1) to comparison with the classical hypergeometric series

$$
{ }_{p} \mathcal{F}_{q}\left(\alpha_{1}, \ldots, \alpha_{p} ; \beta_{1}, \ldots, \beta_{q} ; \xi\right)=\sum_{d=0}^{\infty} \frac{\left(\alpha_{1}\right)_{d} \cdots\left(\alpha_{p}\right)_{d}}{\left(\beta_{1}\right)_{d} \cdots\left(\beta_{q}\right)_{d}} \frac{\xi^{d}}{d !}
$$

which is an entire function of $\xi$ when $p \leq q$, has radius of convergence 1 when $p=q+1$, and (unless a denominator coefficient is a negative integer), diverges for all $\xi \neq 0$ if $p>q$. For this purpose we first prove a coarse estimate (6.5.1) from which part (1) of the theorem is an easy corollary. We also make an exact calculation (6.8.1) from which part (3) and the divergence in part (2) are straightforward consequences. However, our proof of $(2)$ is more involved, resulting from the fact that the estimate (6.5.1), as it applies when $p=q+1$, yields convergence only in a smaller domain $\{s \in S:\|s\|<1 / \bar{n}\}$. Here, and throughout this section, $\bar{n}=n / \eta$ with $\eta$ given by (1.2.1); that is, $\bar{n}=n$ over $\mathbf{R}$ or $\mathbf{C}$, and $\bar{n}=2 n$ over $\mathbf{H}$. To complete part (2), we give a second representation (6.11.1) of ${ }_{p+1} F_{p}$ as an Eulertype integral from which the convergence on the larger domain $\{s \in S:\|s\|<1\}$ follows by an abstract extension theorem (Lemma 6.10). In this connection we wish to thank J. Polking and H. Rossi for sharing with us their expertise on the subject of domains of holomorphy.

6.5 Lemma. Let $|m|=d$. Then

$$
\left|Z_{m}(s)\right| \leq \bar{n}^{d}\|s\|^{d}
$$

for all $s \in S$. 
PROOF. This inequality follows from the Binet-Cauchy theorem, the integral representation (4.8.2) for the highest weight vector $q_{m} \in \mathcal{P}^{m}(S)$, and the definition (5.3.1) of $Z_{m}$. Let $D$ denote the determinant of a matrix, and recall that $\Delta=D^{\eta}$ is a polynomial on $S$. For $j=1, \ldots, n$ we set $J=j / \eta$. If $x$ is any complex $\bar{n} \times \bar{n}$ matrix, form the $J \times J$ minor $D_{l_{1}, \ldots, l_{J}}^{i_{1}, \ldots, i_{J}}(x)$ from rows $i_{1}, \ldots, i_{J}$ and columns $l_{1}, \ldots, l_{J}$ of $x$, where $1 \leq i_{1}<i_{2}<\cdots<i_{J} \leq \bar{n}$ and $1 \leq l_{1}<l_{2}<\cdots<l_{J} \leq \bar{n}$. We set

$$
D_{J}(x)=D_{1,2, \ldots, J}^{1,2, \ldots, J}(x) \text {. }
$$

The Binet-Cauchy theorem states that for any matrices $x$ and $y$

$$
D_{l_{1}, \ldots, l_{J}}^{i_{1}, \ldots, i_{J}}(x y)=\sum_{h_{1}<\cdots<h_{J}} D_{h_{1}, \ldots, h_{J}}^{i_{1}, \ldots, i_{J}}(x) D_{l_{1}, \ldots, l_{J}}^{h_{1}, \ldots, h_{J}}(y) .
$$

In particular, if $k \in K$, so $k k^{*}=1$, then

$$
\sum_{l_{1}<\cdots<l_{J}}\left|D_{l_{1}, \ldots, l_{J}}^{1, \ldots, J}(k)\right|^{2}=1
$$

for all $j=1, \ldots, n$. Clearly, for a diagonal $\bar{n} \times \bar{n}$ complex matrix $\lambda$

$$
D_{l_{1}, \ldots, l_{J}}^{i_{1}, \ldots, i_{J}}(\lambda)=0
$$

if $\left(i_{1}, \ldots, i_{J}\right) \neq\left(l_{1}, \ldots, l_{J}\right)$. Thus,

$$
\begin{aligned}
\left|D_{J}\left(k^{*} \lambda k\right)\right| & =\left|\sum_{i_{1}<\cdots<i_{J}} \sum_{l_{1}<\cdots<l_{J}} D_{i_{1}, \ldots, i_{J}}^{1, \ldots, J}\left(k^{*}\right) D_{l_{1}, \ldots, l_{J}}^{i_{1}, \ldots, i_{J}}(\lambda) D_{1, \ldots, J}^{l_{1}, \ldots, l_{J}}(k)\right| \\
& =\left|\sum_{i_{1}<\cdots<i_{J}} D_{i_{1}, \ldots, i_{J}}^{1, \ldots, J}\left(k^{*}\right) D_{1, \ldots, J}^{i_{1}, \ldots, i_{J}}(k) \lambda_{i_{1}} \cdots \lambda_{i_{J}}\right| \\
& \leq\|\lambda\|^{J} \sum_{i_{1}<\cdots<i_{J}}\left|D_{i_{1}, \ldots, i_{J}}^{1, \ldots, J}(k)\right|^{2}=\|\lambda\|^{J}
\end{aligned}
$$

and

$$
\left|\Delta_{j}\left(k^{*} \lambda k\right)\right|=\left|D_{J}\left(k^{*} \lambda k\right)\right|^{\eta} \leq\|\lambda\|^{j}
$$

for all $j=1, \ldots, n$. If we diagonalize $s \in S$ as $s=k^{*} \lambda k$, then by (4.8.2)

$$
\begin{aligned}
\left|f_{m}(s)\right| & =\left|\int_{K} q_{m}\left(k^{*} \lambda k\right) d k\right| \leq \int_{K}\left|q_{m}\left(k^{*} \lambda k\right)\right| d k \\
& =\int_{K}\left|\Delta\left(k^{*} \lambda k\right)\right|^{m_{n}} \prod_{j=1}^{n-1}\left|\Delta_{j}\left(k^{*} \lambda k\right)\right|^{m_{j}-m_{j+1}} d k \\
& \leq\|\lambda\|^{d}=\|s\|^{d} .
\end{aligned}
$$

By (5.3.1) and (5.3.3), $Z_{m}(s)=Z_{m}(1) f_{m}(s)$ and

$$
0<Z_{m}(1)<\sum_{|m|=d} Z_{m}(1)=(\operatorname{tr} 1)^{d}=\bar{n}^{d}
$$

which establishes inequality (1). 
6.6 The comparison test for convergence. Let

$$
\begin{array}{rlrl}
\alpha_{i j} & =\alpha_{i}-(j-1) \nu / 2, & i & =1, \ldots, p, \\
\beta_{i j}=\beta_{i}-(j-1) \nu / 2, & j & =1, \ldots, q,
\end{array}
$$

and set

$$
{ }_{p} \mathcal{F}_{q}^{(j)}(\xi)={ }_{p} \mathcal{F}_{q}\left(\alpha_{1 j}, \ldots, \alpha_{p j} ; \beta_{1 j}, \ldots, \beta_{q j} ; \xi\right)
$$

for $\xi \in \mathbf{C}$ and $j=1, \ldots, n$. With $d=|m|$, we have

$$
1 / d ! \leq 1 / m_{1} ! m_{2} ! \cdots m_{n} !
$$

and by (6.5.1)

$$
\begin{aligned}
& \sum_{d=0}^{\infty} \sum_{|m|=d}\left|\frac{\left[\alpha_{1}\right]_{m} \cdots\left[\alpha_{p}\right]_{m}}{\left[\beta_{1}\right]_{m} \cdots\left[\beta_{q}\right]_{m}} \frac{Z_{m}(s)}{d !}\right| \\
& \quad \leq \sum_{d=0}^{\infty} \sum_{|m|=d}\left|\frac{\left[\alpha_{1}\right]_{m} \cdots\left[\alpha_{p}\right]_{m}}{\left[\beta_{1}\right]_{m} \cdots\left[\beta_{q}\right]_{m}}\right| \frac{\bar{n}^{d}\|s\|^{d}}{m_{1} ! \cdots m_{n} !} \\
& \quad \leq \prod_{j=1}^{n}\left\{\sum_{m_{j}=0}^{\infty}\left|\frac{\left(\alpha_{1 j}\right)_{m_{j}}\left(\alpha_{2 j}\right)_{m_{j}} \cdots\left(\alpha_{p j}\right)_{m_{j}}}{\left(\beta_{1 j}\right)_{m_{j}}\left(\beta_{2 j}\right)_{m_{j}} \cdots\left(\beta_{q j}\right)_{m_{j}}}\right| \frac{(\bar{n}\|s\|)^{m_{j}}}{m_{j} !}\right\}
\end{aligned}
$$

which compares the absolute convergence of ${ }_{p} F_{q}(s)$ with that of $\prod_{j=1}^{n}{ }_{p}^{\mathcal{F}_{q}^{(j)}}(\xi)$ for $\xi=\bar{n}\|s\|$. Thus, for $p \leq q$ the series ${ }_{p} F_{q}(s)$ converges absolutely for all $s \in S$; and for $p=q+1$, the series ${ }_{p+1} F_{p}(s)$ converges for $\|s\|<1 / \bar{n}$.

6.7 REMARKS. We will consider the hypergeometric series (6.1.1) as a function of the parameters as well as the argument. To minimize the number of variables involved, note that a $K$-invariant real-analytic function on $S$ is determined uniquely by restriction to the positive diagonal matrices. Similarly, a $K^{\mathrm{C}}$-invariant holomorphic function on $S^{\mathbf{C}}$ is determined by restriction to the complex diagonal matrices. More formally, let $W_{n}$ be the symmetric group on $n$ letters. Then the algebra of $K^{\mathbf{C}}$-invariant holomorphic functions on $S^{\mathbf{C}}$ is isomorphic (by restriction to the diagonal) to the algebra of $W_{n}$-invariant holomorphic functions on $\mathbf{C}^{n}[\mathbf{3}, \mathbf{2 1}]$. In particular, ${ }_{p} F_{q}(s)={ }_{p} F_{q}(\lambda)$ where $\lambda$ is the diagonal matrix in $\mathbf{F}^{n \times n}$, the diagonal entries of which are the eigenvalues of $s$. Thus, we can, and frequently shall, view the hypergeometric function as a symmetric function of the eigenvalues $\lambda_{1}, \ldots, \lambda_{n}$. A fortiori, by analytic continuation in these variables, the hypergeometric function becomes a function of $n$ complex variables $\lambda_{1}, \ldots, \lambda_{n}$. When $\mathbf{F}=\mathbf{H}$, we must remember that each eigenvalue $\lambda_{i}$ of $s \in S$ appears with multiplicity two. Thus, $\lambda=\operatorname{diag}\left(\lambda_{1}, \lambda_{1}, \lambda_{2}, \lambda_{2}, \ldots, \lambda_{n}, \lambda_{n}\right)$ when $\mathbf{F}=\mathbf{H}$, whereas $\lambda=\operatorname{diag}\left(\lambda_{i}, \ldots, \lambda_{n}\right)$ when $\mathbf{F}=\mathbf{R}$ or $\mathbf{F}=\mathbf{C}$. We will choose convenience over precision of expression, and by an abuse of notation write

$$
\lambda=\left(\lambda_{1}, \lambda_{2}, \ldots, \lambda_{n}\right), \quad \lambda_{i} \in \mathbf{C},
$$

for both a variable in $\mathbf{C}^{n}$ as well as a diagonal matrix with $\bar{n}=n / \eta$ diagonal entries $\lambda_{1}, \ldots, \lambda_{n}$ each counted with multiplicity $1 / \eta$. Thus, the argument in (6.6) shows that when we normalize the hypergeometric function to avoid poles of the 
coefficients, we obtain a mapping

$$
\left(\alpha_{1}, \ldots, \alpha_{p} ; \beta_{1}, \ldots, \beta_{q} ; \lambda\right) \mapsto\left[\prod_{i=1}^{n} \Gamma_{n}\left(\beta_{i}\right)\right]_{p}^{-1} F_{q}\left(\alpha_{1}, \ldots, \alpha_{p} ; \beta_{1}, \ldots, \beta_{q} ; \lambda\right)
$$

that for $p \leq q$ is an entire holomorphic function on $\mathbf{C}^{p+q} \times \mathbf{C}^{n}$, and for $p=q+1$ is a holomorphic function on $\mathbf{C}^{2 p-1} \times D_{1 / \bar{n}}$, where $D_{\varepsilon}$ denotes the polydisc in $\mathbf{C}^{n}$ of radius $\varepsilon$.

6.8 LEMMA. For any nonnegative integer $d$, let $(d)$ be the $n$-tuple $(d)=$ $(d, 0, \ldots, 0)$.

(i) If $s \in S$ has eigenvalues $\lambda_{1}, \ldots, \lambda_{n}$ then

$$
Z_{(d)}(s)=\frac{d !}{(\nu / 2)_{d}} \sum_{i_{1}+\cdots+i_{n}=d} \prod_{j=1}^{n} \frac{(\nu / 2 \eta)_{i_{j}} \lambda_{j}^{i_{j}}}{i_{j} !} .
$$

In (1), $i_{1}, \ldots, i_{n}$ are unordered, nonnegative integers which sum to $d$.

(ii) For $1 \leq r \leq n$, denote by $1_{r}$ the $n \times n$ diagonal matrix with eigenvalues $\lambda_{1}=\cdots=\lambda_{r}=1$ and $\lambda_{r+1}=\cdots=\lambda_{n}=0$. Then

$$
Z_{(d)}\left(1_{r}\right)=\frac{(\nu r / 2 \eta)_{d}}{(\nu / 2)_{d}}
$$

ProOF. From (6.2.2) and the convergence on $\|s\|<1 / \bar{n}$,

$$
{ }_{1} F_{0}(\alpha ; s)=\sum_{d=0}^{\infty} \sum_{|m|=d}[\alpha]_{m} \frac{Z_{m}(s)}{d !}=\Delta(1-s)^{-\alpha}
$$

for all $\alpha \in$ C. By (5.6.3) with $\alpha=\nu / 2$ and $|m|=d$,

$$
\left[\frac{\nu}{2}\right]_{m}=\prod_{j=1}^{n}\left(\frac{(2-j) \nu}{2}\right)_{m_{j}}= \begin{cases}(\nu / 2)_{d}, & \text { when } m=(d) \\ 0, & \text { otherwise. }\end{cases}
$$

Thus, substitute $\alpha=\nu / 2$ and $s=\xi \lambda$ in (3), where $\xi \in \mathbf{R}, \lambda=\left(\lambda_{1}, \ldots, \lambda_{n}\right)$ and $\|\xi \lambda\|<1 / \bar{n}$. We obtain

$$
\Delta(1-\xi \lambda)^{-\nu / 2}=\sum_{d=0}^{\infty} \frac{(\nu / 2)_{d} \xi^{d} Z_{(d)}(\lambda)}{d !}
$$

But we also have

$$
\begin{aligned}
(1-\xi \lambda)^{-\nu / 2} & =\prod_{j=1}^{n}\left(1-\xi \lambda_{j}\right)^{-\nu / 2 \eta}=\prod_{j=1}^{n}\left\{\sum_{i_{j}=0}^{\infty} \frac{(\nu / 2 \eta)_{i_{j}} \xi^{i_{j}} \lambda_{j}^{i_{j}}}{i_{j} !}\right\} \\
& =\sum_{d=0}^{\infty} \xi^{d} \sum_{i_{1}+\cdots+i_{n}=d} \prod_{j=1}^{n} \frac{(\nu / 2 \eta)_{i_{j}} \lambda_{j}^{i_{j}}}{i_{j} !}
\end{aligned}
$$

Then (1) follows on comparing the coefficient of $\xi^{d}$ in (4) and (5). Finally, (2) follows as a special case of (1). 
6.9 Comparison test for divergence. From the above lemma, if $\xi \in \mathbf{R}, \xi \neq 0$, and $1 \leq r \leq n$, then

$$
\begin{array}{r}
\sum_{d=0}^{\infty} \sum_{|m|=d}\left|\frac{\left[\alpha_{1}\right]_{m}\left[\alpha_{2}\right]_{m} \cdots\left[\alpha_{p}\right]_{m}}{\left[\beta_{1}\right]_{m}\left[\beta_{2}\right]_{m} \cdots\left[\beta_{q}\right]_{m}}\right| \frac{\left|Z_{m}\left(\xi 1_{r}\right)\right|}{d !} \\
\geq \sum_{d=0}^{\infty}\left|\frac{\left[\alpha_{1}\right]_{(d)} \cdots\left[\alpha_{p}\right]_{(d)}}{\left[\beta_{1}\right]_{(d)} \cdots\left[\beta_{q}\right]_{(d)}}\right| \frac{|\xi|^{d} Z_{(d)}\left(1_{r}\right)}{d !} \\
=\sum_{d=0}^{\infty}\left|\frac{\left(\alpha_{1}\right)_{d} \cdots\left(\alpha_{p}\right)_{d}}{\left(\beta_{1}\right)_{d} \cdots\left(\beta_{q}\right)_{d}}\right| \frac{(\nu r / 2 \eta)_{d}}{(\nu / 2)_{d}} \frac{|\xi|^{d}}{d !}
\end{array}
$$

which compares the divergence of ${ }_{p+1} \mathcal{F}_{q+1}\left(\alpha_{1}, \ldots, \alpha_{p}, \nu r / 2 \eta ; \beta_{1}, \ldots, \beta_{q}, \nu / 2 ; \xi\right)$ with ${ }_{p} F_{q}\left(\alpha_{1}, \ldots, \alpha_{p} ; \beta_{1}, \ldots, \beta_{q} ; \xi 1_{r}\right)$. Thus, when $p>q$ the series (6.1.1) diverges for all nonzero $s \in S$. Similarly, for $p=q+1$ the series (6.1.1) diverges for $\|s\|>1$.

The proof of Theorem 6.3 is nearly complete. All that remains is to prove the convergence of ${ }_{p+1} F_{p}$ on the full domain $\|s\|<1$. In fact, we prove a stronger version contained in Proposition 6.11 below.

6.10 Lemma. Let $X=X_{1} \times X_{2}$ where $X_{1}=\mathbf{C}^{i}$ and $X_{2}=\mathbf{C}^{j}$ for some dimensions $i$ and $j$. Let $\Omega_{1}$ and $\Omega_{2}$ be the domains $X_{1} \times D_{a}$ and $U \times D_{b}$ in $X$, respectively, where $\mathcal{U}$ is a domain in $X_{1}$ and $D_{a}, D_{b}$ are polydiscs (about the origin as center) in $X_{2}$ with $0<a<b$. If $f$ is a function holomorphic on $\Omega_{1} \cup \Omega_{2}$, then it extends to a function holomorphic on $X_{1} \times D_{b}$.

Proof. If necessary, choose $x_{0} \in X_{1}$ such that $x_{0}+\mathcal{U}$ contains a polydisc $D_{\varepsilon}$ (about the origin as center) in $X_{1}$, and let $h\left(x_{0}+x, y\right)=f(x, y)$ for $(x, y)$ in the domain of $f$. Then the union of $\Omega_{1}$ and $D_{\varepsilon} \times D_{b}$ is a Reinhardt domain, to which the extension theorem [17, Theorem 2.4.6] applies. Thus, $h$ extends to a holomorphic function on the logarithmically convex hull of the union of $\Omega_{1}$ and $D_{\varepsilon} \times D_{b}$, which in this case coincides with $X_{1} \times D_{b}$. Clearly, $f$ also extends to $X_{1} \times D_{b}$.

6.11 Proposition. For $p=q+1$, the mapping (6.7.2) defines a function that is holomorphic on $\mathbf{C}^{2 p-1} \times D_{1}$.

PROOF. We argue by induction on $p$. In general, we know that the function

$$
\begin{aligned}
& f_{p}\left(\alpha_{1}, \ldots, \alpha_{p} ; \beta_{1}, \ldots, \beta_{p-1} ; \lambda\right) \\
& \quad=\left[\prod_{j=1}^{n} \Gamma_{n}\left(\beta_{j}\right)\right]^{-1}{ }_{p} F_{p-1}\left(\alpha_{1}, \ldots, \alpha_{p} ; \beta_{1}, \ldots, \beta_{p-1} ; \lambda\right)
\end{aligned}
$$

is holomorphic on $\mathbf{C}^{2 p-1} \times D_{1 / \bar{n}}$. Let $p=1$. Then $f_{0}(\alpha ; \lambda)=\Delta(1-\lambda)^{-\alpha}$, and $f_{0}$ is seen to be holomorphic on $\mathbf{C} \times D_{1}$. As induction hypothesis, assume that $f_{p}$ is holomorphic on $\mathbf{C}^{2 p-1} \times D_{1}$. Consider $f_{p+1}$. By (5.10) and Fubini's theorem

$$
\int_{0<r<1} \Delta(r)^{\alpha_{p+1}} \Delta(1-r)^{\beta_{p}-\alpha_{p+1}-\theta}{ }_{p} F_{p-1}\left(\alpha_{1}, \ldots, \alpha_{p} ; \beta_{1}, \ldots, \beta_{p-1} ; r \lambda\right) d_{*} r
$$

$$
=\frac{\Gamma_{n}\left(\alpha_{p+1}\right) \Gamma_{n}\left(\beta_{p}-\alpha_{p+1}\right)}{\Gamma_{n}\left(\beta_{p}\right)}{ }_{p+1} F_{p}\left(\alpha_{1}, \ldots, \alpha_{p+1} ; \beta_{1}, \ldots, \beta_{p} ; \lambda\right)
$$


for $\operatorname{Re}\left(\beta_{p}\right)>\operatorname{Re}\left(\alpha_{p+1}\right)+(n-1) \nu / 2>(n-1) \nu$ and $\|\lambda\|<1$. Thus,

$$
\begin{aligned}
& f_{p+1}\left(\alpha_{1}, \ldots, \alpha_{p+1} ; \beta_{1}, \ldots, \beta_{p} ; \lambda\right) \\
& =\left[\Gamma_{n}\left(\alpha_{p+1}\right) \Gamma_{n}\left(\beta_{p}-\alpha_{p+1}\right)\right]^{-1} \int_{0<r<1} \Delta(r)^{\alpha_{p+1}} \Delta(1-r)^{\beta_{p}-\alpha_{p+1}-\theta} \\
& \times f_{p}\left(\alpha_{1}, \ldots, \alpha_{p} ; \beta_{1}, \ldots, \beta_{p-1} ; r \lambda\right) d_{*} r
\end{aligned}
$$

is holomorphic on a domain of the form $U \times D_{1}$ in $\mathbf{C}^{2 p+1} \times \mathbf{C}^{n}$, where $\mathcal{U}$ is a domain in $\mathbf{C}^{2 p+1}$. By Lemma $6.10, f_{p}$ is then holomorphic on $\mathbf{C}^{2 p+1} \times D_{1}$. This completes the induction and also the proof of Theorem 6.3.

6.12 EXAMPLE. The special case $p=2$ in Proposition 6.11 describes the analogue

$$
{ }_{2} F_{1}(a, b ; c ; s)=\sum_{d=0}^{\infty} \sum_{|m|=d} \frac{[\alpha]_{m}[b]_{m}}{[c]_{m}} \frac{Z_{m}(s)}{d !}
$$

of the classical Gaussian hypergeometric function. In the complex case this has also been studied by Louck and Biedenharn [22]. By (6.11.1), we have the Euler formula

(2) ${ }_{2} F_{1}(a, b ; c ; s)=\frac{\Gamma_{n}(c)}{\Gamma_{n}(b) \Gamma_{n}(c-b)} \int_{0<r<1} \Delta(r)^{b} \Delta(1-r)^{c-b-\theta} \Delta(1-r s)^{-a} d_{*} r$

for $\operatorname{Re} c>\operatorname{Re} b+(n-1) \nu / 2>(n-1) \nu$ and $\|s\|<1$, where $\theta$ is given by (1.6.4).

6.13 THEOREM (LAPLACE TRANSFORMS OF HYPERGEOMETRIC FUNCTIONS). Suppose $p \leq q$ and $\operatorname{Re}\left(\alpha_{p+1}\right)>(n-1) \nu / 2$. Then

$$
\begin{gathered}
\frac{1}{\Gamma_{n}\left(\alpha_{p+1}\right)} \int_{P} e_{p}^{-\operatorname{tr}(r z)} F_{q}\left(\alpha_{1}, \ldots, \alpha_{p} ; \beta_{1}, \ldots, \beta_{q} ; r\right) \Delta(r)^{\alpha_{p+1}} d_{*} r \\
=\Delta(z)^{-\alpha_{p+1}}{ }_{p+1} F_{q}\left(\alpha_{1}, \ldots, \alpha_{p+1} ; \beta_{1}, \ldots, \beta_{q} ; z^{-1}\right)
\end{gathered}
$$

When $p<q$, the integral in (1) converges absolutely for all $z$ in the right halfplane $\Phi$. When $p=q$, the integral converges absolutely for all $z \in S^{\mathbf{C}}$ such that $0<(\operatorname{Re} z)^{-1}<1$.

ProOF. To simplify notation, write $\alpha_{p+1}=\alpha, \operatorname{Re}(\alpha)=a, z=x+i y$ with $x, y \in S$, and let $c_{m}$ be the coefficient of $Z_{m}$ in ${ }_{p} F_{q}$. We assume $a>(n-1) \nu / 2$ and $x \in P$. The integrand in (1) is

$$
F(r)=\sum_{m} c_{m} e^{-\operatorname{tr}(r z)} \Delta(r)^{\alpha} Z_{m}(r) .
$$

Formal term by term integration, based upon Theorem 5.9, yields formula (1). Therefore, it suffices to verify the hypotheses of Fubini's theorem; namely, the series 
in (2) is absolutely integrable. By (5.9) and the monotone convergence theorem,

$$
\begin{aligned}
& \int_{P} \sum_{m}\left|c_{m} e^{-\operatorname{tr}(r z)} \Delta(r)^{\alpha} Z_{m}(r)\right| d_{*} r \\
& =\int_{P}\left\{\sum_{m}\left|c_{m}\right| e^{-\operatorname{tr}(r x)} \Delta(r)^{a} Z_{m}(r)\right\} d_{*} r \\
& =\sum_{m}\left|c_{m}\right| \int_{P} e^{-\operatorname{tr}(r x)} \Delta(r)^{a} Z_{m}(r) d_{*} r \\
& =\Gamma_{n}(a) \Delta(x)^{-a} \sum_{m}\left|c_{m}\right|[a]_{m} Z_{m}\left(x^{-1}\right)
\end{aligned}
$$

We recognize this latter series as expressing the absolute convergence of ${ }_{p+1} F_{q}\left(x^{-1}\right)$. When $p<q$, this series is finite for all $x^{-1}$; hence, when $p<q$, (1) holds for all $z \in P+i S=\Phi$. When $p=q$, the above series converges only when $\left\|x^{-1}\right\|<1$; hence, for $p=q,(1)$ is valid for all $z \in P+i S=\Phi$ such that $\left\|(\operatorname{Re} z)^{-1}\right\|<1$, or equivalently $0<(\operatorname{Re} z)^{-1}<1$.

\section{REFERENCES}

1. S. Bochner, Bessel functions and modular relations of higher type and hyperbolic differential equations, Comm. Sem. Math. de l'Univ. Lund, Tome supplementaire (1952), 12-20.

2. A. G. Constantine, Some noncentral distribution problems in multivariate analysis, Ann. Math. Statist. 34 (1963), 1270-1285.

3. J. Dadok and V. Kac, Polar representations, J. Algebra 92 (1985), 504-524.

4. B. E. Eichinger, An approach to distribution functions for Gaussian molecules, Macromolecules 10 (1977), 671-675.

5. R. Godement, $A$ theory of spherical functions. I, Trans. Amer. Math. Soc. 73 (1952), 496-556.

6. S. G. Gindikin, Analysis on homogeneous spaces, Russian Math. Surveys 19 (1964), 1-90.

7. K. I. Gross and R. A. Kunze, Fourier decompositions of certain representations, Analysis and Geometry of Symmetric Spaces, Dekker, New York, 1972.

8. , Bessel functions and representation theory. I, J. Funct. Anal. 22 (1976), 73-105.

9. __ Bessel functions and representation theory. II: Holomorphic discrete series and metaplectic representations, J. Funct. Anal. 25 (1977), 1-49.

10. __ Finite dimensional induction and new results on invariants for classical groups. I, Amer. J. Math. 106 (1984), 893-974.

11. _ Finite dimensional induction. II: General reciprocity theorems, J. Reine Angew. Math. 369 (1986), 14-20.

12. K. I. Gross and D. St. P. Richards, Special functions of matrix argument. II (in preparation).

13. S. Helgason, Differential geometry, Lie groups and symmetric spaces, Academic Press, New York, 1979.

14. , Groups and geometric analysis, Academic Press, New York, 1984.

15. C. S. Herz, Bessel functions of matrix argument, Ann. of Math. (2) 61 (1955), 474-523.

16. W. J. Holman III, Generalized Bessel functions and the representation theory of $\mathcal{U}(2) \odot \mathrm{C}^{2 \times 2}, \mathrm{~J}$. Math. Phys. 21 (1980), 1977-2010.

17. L. Hormander, An introduction to complex analysis in several variables, Van Nostrand, Princeton, N. J., 1966.

18. L. K. Hua, Harmonic analysis of functions of several complex variables in the classical domains, Transl. Math. Monographs, vol. 6, Amer. Math. Soc., Providence, R. I., 1963.

19. A. T. James, Distributions of matrix variates and latent roots derived from normal samples, Ann. Math. Statist. 35 (1964), 475-501.

20. K. Johnson, On a ring of invariant polymomials on a hermitian symmetric space, J. Algebra 67 (1980), 72-81. 
21. B. Kostant and S. Rallis, Orbits and representations associated with symmetric spaces, Amer. J. Math. 93 (1971), 753-809.

22. J. D. Louck and L. C. Biedenharn, A generalization of the Gauss hypergeometric function, J. Math. Anal. Appl. 59 (1977), 423-431.

23. R. J. Muirhead, Aspects of multivariate statistical theory, Wiley, New York, 1983.

24. D. Richards, Solution to problem 84-1: An integral on SO(3), SIAM Rev. 27 (1985), 81-82.

25. W. Schmid, Die Randwerte holomorpher Funktionen auf hermitesch symmetrischen Raümen, Invent. Math. 9 (1969), 61-80.

26. A. Selberg, Harmonic analysis and discontinuous groups in weakly symmetric Riemannian spaces with applications to Dirichlet spaces, J. Indian Math. Soc. 20 (1956), 47-87.

27. G. Shimura, Confluent hypergeometric functions on tube domains, Math. Ann. 260 (1982), 269302.

28. C. L. Siegel, Über die analytische Theorie der quadratischen Formen. I, Ann. of Math. (2) 36 (1935), 527-608.

29. A. Takemura, Zonal polymomials, Inst. Math. Statist., Monograph Series, vol. 4, California, 1985.

30. M. Takeuchi, Polymomial representations associated with symmetric bounded domains, Osaka J. Math. 10 (1973), 441-475.

31. A. Terras, Special functions for the symmetric space of positive matrices, SIAM J. Math. Anal. 16 (1985), 620-640.

32. __ Harmonic analysis on symmetric spaces and applications. I, Springer-Verlag, New York, 1985.

33. T. T. That, Lie group representations and harmonic polymomials of a matrix variable, Trans. Amer. Math. Soc. 216 (1976), 1-46.

34. H. Weyl, The classical groups, Princeton Univ. Press, Princeton, N. J., 1949.

Division of Mathematical Sciences, National Science foundation, WashINGTON, D.C. 20550

Department of Statistics, University of North Carolina at Chapel Hill, Chapel Hill, North Carolina 27514 